\title{
CFD Implementation of a novel carbon-phenolic-in-air chemistry model for atmospheric re-entry
}

\author{
Alexandre Martin* and Iain D. Boyd ${ }^{\dagger}$ \\ Department of Aerospace Engineering, The University of Michigan, Ann Arbor, MI, 48109, USA
}

\begin{abstract}
Recent and future re-entry vehicle designs use ablative material as the main component of the heat shield of their thermal protection systems. In order to properly predict the behavior of the vehicle, it is imperative to take into account the gases produced by the ablation process when modeling the reacting flow environment. In the case of charring ablators, where an inner resin is pyrolyzed at a relatively low temperature, the composition of the gas expelled in the boundary layer is complex and might lead to thermal chemical reactions that cannot be captured with simple flow chemistry models. In order to obtain better predictions, a proper gas flow chemistry model needs to be included in the CFD calculations. Recent calculations showed that extensive differences are found in boundary layer composition and heat fluxes, both convective and radiative, when previously published models were used on a realistic problem. Recently, a more complete model was proposed, which includes an extensive set of kinetic rates, taken from the combustion community. Using this model, CFD calculations of the Stardust re-entry are presented. The results clearly demonstrate the need to account for many more species in the flow field than the ones that are expected to be present at the surface.
\end{abstract}

\section{Nomenclature}

Symbols

A Pre-exponential factor

$\mathrm{B}^{\prime} \quad$ Non dimensional ablation rate

D Mass diffusion coefficient

$k \quad$ Kinetic rate

$\dot{m} \quad$ Mass flow rate

$n \quad$ Pre-exponential temperature power

$p \quad$ Pressure

ppm Parts per million

Subscripts

c Char

$g \quad$ Gas blown

nc Next to the wall

$p \quad$ Pyrolysis

$r \quad$ Reaction, Rotational
$S \quad$ Non dimensional sensitivity

$T$ Temperature

$T_{a} \quad$ Activation temperature

$U, v \quad$ Velocity

$X \quad$ Sensitivity variable

$Y \quad$ Mass fraction

$\eta \quad$ Distance normal to the wall

$\rho \quad$ Density

*Research Associate, AIAA Senior Member; currently Assistant Professor, Department of Mechanical Engineering, University of Kentucky, Lexington, KY, 40506, USA

$\dagger$ James E. Knott Professor of Engineering, Associate Fellow AIAA. 


\section{Introduction}

The Thermal Protection System (TPS) of a re-entry vehicle is one of the key components of its design. The materials used for the TPS can be classified into two main categories: ablative materials, such as the ones used on the Apollo missions, and non-ablative materials, such as the ceramic tiles used on the Space Shuttle. The former can also be divided into two sub-categories: charring (also know as pyrolyzing) and non-charring ablators. The theory behind the use of ablators is quite simple: the energy absorbed by the removal of material from the surface is not used to heat the TPS, thus keeping the vehicle at a relatively "cold" temperature. In the case of charring ablators, the ablative material is a resin which fills the pores of a carbon matrix. Although the matrix might ablate, it usually does not, thus preserving the original geometry of the aerodynamic surface during re-entry.

In order to properly model the heat rates at the surface of the vehicle, the ablating boundary condition must take into account many phenomena: surface recession, wall temperature, blowing rates, gas composition, surface chemistry, etc. However, to account for the effects of the pyrolysis gas on the vehicle, the chemistry model of the flow field must include the reactions associated with the presence of this gas. Because ablation coupling is becoming an increasingly important research topic, ${ }^{1-7}$ the development of an accurate, yet usable, chemistry model is of great importance. Models have been proposed in the past ${ }^{8-10}$ but important reactions were not included, and some of the reaction rates were inappropriate or simply outdated.

Recently, a more complete model was proposed, ${ }^{11}$ which includes an extensive of set kinetic rates, taken from the combustion community. The model was reduced using 0-D sensitivity analysis on a parameter space relevant to the re-entry conditions that such a material would be exposed to. It was established that such a model was necessary to study carbon/phenolic TPS through a review of past models, which gave a wide range of results, especially when radiative heat transfer calculations were performed. ${ }^{12}$

This paper proposes to proceed to the next step by integrating the reduced model in the hypersonic Computational Fluid Dynamics (CFD) code LeMANS. As a test case, the Stardust re-entry vehicle is used. The trajectory points chosen are the ones corresponding to the Echelle period, which ranges from an altitude of $81 \mathrm{~km}$ to an altitude of $69 \mathrm{~km}$. This test-case is chosen so that the computed results can be processed through a radiation code, namely, NEQAIR (version 99d) ${ }^{13}$ and then compared to the experimental data gathered by the Echelle instrument.

\section{Chemistry model}

\section{A. Selection of species and chemical reactions}

Previous results ${ }^{12}$ showed a need to develop an exhaustive, validated and properly reduced chemistry model for ablating carbon phenolic in air. Such a model was recently developed and presented in Ref. 11. The model was constructed by carefully selecting the species important to carbon-phenolic ablation in air. First, all the usual ionizing air species are included:

$$
\mathrm{N}_{2}, \mathrm{O}_{2}, \mathrm{NO}, \mathrm{N}, \mathrm{O}, \mathrm{N}_{2}^{+}, \mathrm{O}_{2}^{+}, \mathrm{NO}^{+}, \mathrm{N}^{+}, \mathrm{O}^{+}, \mathrm{e}
$$

Assuming that the ablation and pyrolysis gas is in equilibrium at the surface of the vehicle, it is possible to evaluate the important ablation species. In order to do so, the re-entry trajectory of the Stardust vehicle and proposed trajectories of the CEV are used. The method of analysis for the thermal protection system begins with computing the flow field over a discrete set of points along the estimated flight trajectory to sufficiently capture the heat pulse. High fidelity solutions are computed at several points in the trajectory with the CFD code DPLR. ${ }^{14}$ For Stardust, these discrete solutions are interpolated (using engineering relationships) in time along the trajectory. The heat transfer coefficient, the surface pressure and the freestream enthalpy are the environment inputs calculated for each body point along the entire trajectory and used in the material response code, FIAT. ${ }^{15}$ For the CEV cases, the aerothermodynamic analysis tool CBAero ${ }^{16}$ is used to provide the same input parameters. For selected time steps throughout the analyzed trajectories, the pressure and the non-dimensionalized ablation rate $\mathrm{B}_{g}^{\prime}$ and $\mathrm{B}_{c}^{\prime}$ values predicted by FIAT are then extracted and input to the Multicomponent Ablation Thermochemistry (MAT) code. ${ }^{17}$ When given this information, MAT calculates back from the $\mathrm{B}^{\prime}$ tables the species mole fractions according to the JANNAF database information corresponding to the wall temperature predicted by FIAT. From these results, trajectory points are chosen so that the widest possible combination of pressure and temperature is represented. Figure 1 shows the parameter space represented by those points. From this analysis, the ablating species at the 


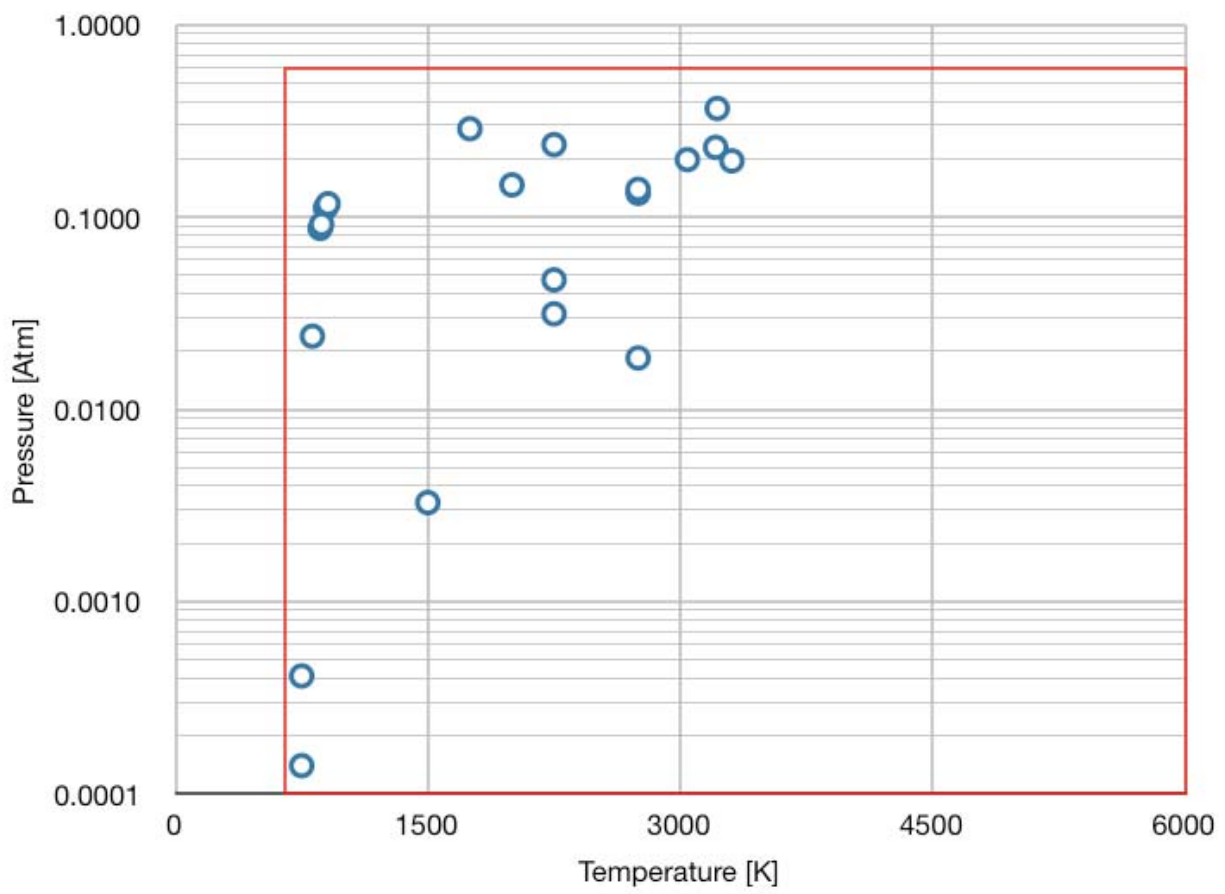

Figure 1. Temperature vs. pressure for various surface locations, trajectory points and re-entry vehicles

surface are:

$$
\mathrm{H}_{2}, \mathrm{CO}, \mathrm{CH}_{4}, \mathrm{H}_{2} \mathrm{O}, \mathrm{CO}_{2}, \mathrm{OH}, \mathrm{C}_{2} \mathrm{H}_{2}, \mathrm{HCN}, \mathrm{C}_{2} \mathrm{H}, \mathrm{C}_{3}, \mathrm{CN}
$$

Since the ablating species are likely to be broken down as they travel through the boundary layer and in the post-shock layer, it is important to include possible reaction paths leading to smaller molecules, as well as species from important reactions. Ionized species also need to be included in the model since charged particles could potentially still reach the boundary layer. Additionally, through associative ionization, electron-impact ionization and, especially, charge exchange reactions, some of the carbon species could be ionized.

Because phenolic vapor, produced by the pyrolysis of carbon-phenolic, is very similar in therms of reactions to a combustion problem, the kinetic rates gathered from that community are used as a basis for the complete model. Because it is accurate, complete, and comprehensive, the GRI-MECH database, ${ }^{18}$ built by The University of California at Berkeley, Stanford University and The University of Texas at Austin, has been chosen. One of the problems with these rates is that they are only valid up to $5000 \mathrm{~K}$, which is not a sufficient temperature range for the gases coming out of the boundary layer. However, since the pyrolysis gases are not expected to enter regions of the flow where higher temperatures are reached (i.e. the shock layer), this assumption is valid for the current application. To account for the gases that are present in high temperature regions, reaction rates relevant to those regions are replaced, mostly by those from Ref. 8 and Ref. 19, which were validated across a wide temperature range.

This methodology results in approximately 55 species and 240 reactions, which is too many for the model to be included in a complex CFD calculation. This model is therefore reduced using the sensitivity analysis software SENKIN, part of the CHEMKIN package. ${ }^{20}$ SENKIN performs a non-linear sensitivity analysis on the rate coefficients, and outputs the results in the form of a normalized maximum sensitivity parameter $S$. For each reaction $r, S$ is computed over time using:

$$
S_{t, r}=\frac{k_{r}}{X_{\max }} \frac{\partial X_{t}}{\partial k_{r}}
$$

where $X_{t}$ represents the parameter on which the sensitivity analysis has been performed at time $t$, and $k_{r}$ is the kinetic rate of reaction $r . X_{\max }$ is chosen as the maximum value of $X_{t}$ over the simulated time. The 
parameters deemed relevant for ablating Earth entry are the temperature and the number density of CN, $\mathrm{CO}, \mathrm{H}_{2} \mathrm{O}$ and $\mathrm{OH}$.

Validation is performed via a zero dimensional analysis. In order to perform a sensitivity analysis, a parameter space based on temperature, pressure and gas composition is defined. For the purpose of the current analysis, temperatures between of $700 \mathrm{~K}$ and $6000 \mathrm{~K}$ are used. For the pressure, a minimum of $1 \times 10^{-4}$ atm and a maximum of 0.5 atm are chosen; these values are based on the re-entry trajectories presented (partially) in Fig. 1. The square in that figure illustrates this parameter space.

From this analysis, the following species are also selected:

$$
\begin{gathered}
\mathrm{H}, \mathrm{NH}, \mathrm{HO}_{2}, \mathrm{H}_{2} \mathrm{O}_{2}, \mathrm{HCO}, \mathrm{C}, \mathrm{C}_{2}, \mathrm{CH}, \mathrm{CH}_{2}, \mathrm{CH}_{3}, \mathrm{NCO}, \mathrm{HNO} \\
\mathrm{CO}^{+}, \mathrm{CN}^{+}, \mathrm{C}^{+}, \mathrm{H}^{+}
\end{gathered}
$$

which brings the reduced model to 38 species. The 158 reactions considered are listed in Table 1; the kinetic rates, $k$, are presented in a modified Arrhenius format, using the following equation:

$$
k=A T^{n} e^{-T_{a} / T}
$$

\begin{tabular}{|c|c|c|c|c|c|}
\hline$\#$ & Reaction & $\mathrm{A}[\mathrm{mol}, \mathrm{cm}, \mathrm{s}]$ & $\mathrm{n}$ & $T_{a}[\mathrm{~K}]$ & References \\
\hline 1 & $2 \mathrm{HO}_{2} \rightleftharpoons \mathrm{O}_{2}+\mathrm{H}_{2} \mathrm{O}_{2}$ & $4.20 \times 10^{14}$ & 0.0 & 6042.7 & 18 \\
\hline \multirow[t]{6}{*}{2} & $2 \mathrm{OH}+\mathrm{M} \rightleftharpoons \mathrm{H}_{2} \mathrm{O}_{2}+\mathrm{M}$ & $2.30 \times 10^{18}$ & -0.9 & -1700.0 & 18 \\
\hline & & $\mathrm{H}_{2}$ enhanced by 2.00 & & & \\
\hline & & $\mathrm{H}_{2} \mathrm{O}$ enhanced by 6.00 & & & \\
\hline & & $\mathrm{CH}_{4}$ enhanced by 2.00 & & & \\
\hline & & $\mathrm{CO}, \mathrm{CO}^{+}$enhanced by 1.50 & & & \\
\hline & & $\mathrm{CO}_{2}$ enhanced by 2.00 & & & \\
\hline 3 & $2 \mathrm{OH} \rightleftharpoons \mathrm{O}+\mathrm{H}_{2} \mathrm{O}$ & $3.57 \times 10^{4}$ & 2.4 & -1062.5 & 18 \\
\hline 4 & $\mathrm{C}+\mathrm{e} \rightleftharpoons \mathrm{C}^{+}+\mathrm{e}+\mathrm{e}$ & $3.7 \times 10^{31}$ & -3.0 & 130720 & 19 \\
\hline 5 & $\mathrm{C}+\mathrm{H}_{2} \rightleftharpoons \mathrm{CH}+\mathrm{H}$ & $4.00 \times 10^{14}$ & 0.0 & 11700.0 & 21 \\
\hline 6 & $\mathrm{C}+\mathrm{N}_{2} \rightleftharpoons \mathrm{CN}+\mathrm{N}$ & $5.24 \times 10^{13}$ & 0.0 & 22600.0 & 22 \\
\hline 7 & $\mathrm{C}+\mathrm{NO} \rightleftharpoons \mathrm{CN}+\mathrm{O}$ & $2.02 \times 10^{14}$ & -0.3 & 0.0 & 23 \\
\hline 8 & $\mathrm{C}+\mathrm{NO} \rightleftharpoons \mathrm{CO}+\mathrm{N}$ & $2.29 \times 10^{13}$ & 0.0 & 0.0 & 23 \\
\hline 9 & $\mathrm{C}+\mathrm{O} \rightleftharpoons \mathrm{CO}^{+}+\mathrm{e}$ & $8.80 \times 10^{8}$ & 1.0 & 33100.0 & 8 \\
\hline 10 & $\mathrm{C}+\mathrm{O}_{2} \rightleftharpoons \mathrm{O}+\mathrm{CO}$ & $5.80 \times 10^{13}$ & 0.0 & 576.0 & 18 \\
\hline 11 & $\mathrm{C}_{2}+\mathrm{C}_{2} \rightleftharpoons \mathrm{C}_{3}+\mathrm{C}$ & $3.20 \times 10^{14}$ & 0.0 & 0.0 & 24 \\
\hline 12 & $\mathrm{C}_{2}+\mathrm{H}_{2} \rightleftharpoons \mathrm{C}_{2} \mathrm{H}+\mathrm{H}$ & $6.60 \times 10^{13}$ & 0.0 & 4030.0 & 24 \\
\hline 13 & $\mathrm{C}_{2}+\mathrm{N}_{2} \rightleftharpoons \mathrm{CN}+\mathrm{CN}$ & $1.50 \times 10^{13}$ & 0.0 & 21000.0 & 25 \\
\hline 14 & $\mathrm{C}_{2} \mathrm{H}+\mathrm{M} \rightleftharpoons \mathrm{C}_{2}+\mathrm{H}+\mathrm{M}$ & $1.74 \times 10^{35}$ & -5.2 & 57400.0 & 24 \\
\hline 15 & $\mathrm{C}_{2} \mathrm{H}+\mathrm{C} \rightleftharpoons \mathrm{C}_{3}+\mathrm{H}$ & $1.00 \times 10^{14}$ & 0.0 & 0.0 & 26 \\
\hline 16 & $\mathrm{C}_{2} \mathrm{H}_{2}+\mathrm{M} \rightleftharpoons \mathrm{C}_{2} \mathrm{H}+\mathrm{H}+\mathrm{M}$ & $6.96 \times 10^{39}$ & -6.1 & 6 to $\operatorname{tn} 30.0$ & 24 \\
\hline 17 & $\mathrm{C}_{2}+\mathrm{M} \rightleftharpoons 2 \mathrm{C}+\mathrm{M}$ & $4.50 \times 10^{18}$ & -1.0 & 70930 & \\
\hline 18 & $\mathrm{CH}+\mathrm{C} \rightleftharpoons \mathrm{C}_{2}+\mathrm{H}$ & $2.00 \times 10^{14}$ & 0.0 & 0.0 & 26 \\
\hline 19 & $\mathrm{CH}+\mathrm{CH} \rightleftharpoons \mathrm{C}_{2} \mathrm{H}+\mathrm{H}$ & $1.50 \times 10^{14}$ & 0.0 & 0.0 & 26 \\
\hline 20 & $\mathrm{CH}+\mathrm{CO}_{2} \rightleftharpoons \mathrm{HCO}+\mathrm{CO}$ & $1.90 \times 10^{14}$ & 0.0 & 7952.1 & 18 \\
\hline 21 & $\mathrm{CH}+\mathrm{M} \rightleftharpoons \mathrm{C}+\mathrm{H}+\mathrm{M}$ & $1.90 \times 10^{14}$ & 0.0 & 33700.0 & 26 \\
\hline 22 & $\mathrm{CH}+\mathrm{N}_{2} \rightleftharpoons \mathrm{HCN}+\mathrm{N}$ & $4.40 \times 10^{12}$ & 0.0 & 11060.0 & 27 \\
\hline 23 & $\mathrm{CH}+\mathrm{O}_{2} \rightleftharpoons \mathrm{O}+\mathrm{HCO}$ & $6.71 \times 10^{13}$ & 0.0 & 0.0 & 18 \\
\hline 24 & $\mathrm{CH}_{2}+\mathrm{C} \rightleftharpoons \mathrm{C}_{2} \mathrm{H}+\mathrm{H}$ & $5.00 \times 10^{13}$ & 0.0 & 0.0 & 26 \\
\hline 25 & $\mathrm{CH}_{2}+\mathrm{CH} \rightleftharpoons \mathrm{C}_{2} \mathrm{H}_{2}+\mathrm{H}$ & $4.00 \times 10^{13}$ & 0.0 & 0.0 & 26 \\
\hline 26 & $\mathrm{CH}_{2}+\mathrm{CH}_{2} \rightleftharpoons \mathrm{C}_{2} \mathrm{H}_{2}+\mathrm{H}+\mathrm{H}$ & $2.00 \times 10^{14}$ & 0.0 & 5530.0 & 28 \\
\hline 27 & $\mathrm{CH}_{2}+\mathrm{CH}_{2} \rightleftharpoons \mathrm{C}_{2} \mathrm{H}_{2}+\mathrm{H}_{2}$ & $1.58 \times 10^{15}$ & 0.0 & 6010.0 & 28 \\
\hline 28 & $\mathrm{CH}_{2}+\mathrm{CH}_{4} \rightleftharpoons \mathrm{CH}_{3}+\mathrm{CH}_{3}$ & $4.30 \times 10^{12}$ & 0.00 & 5050.0 & 29 \\
\hline 29 & $\mathrm{CH}_{2}+\mathrm{H} \rightleftharpoons \mathrm{CH}+\mathrm{H}_{2}$ & $6.03 \times 10^{12}$ & 0.0 & -900.0 & 30 \\
\hline 30 & $\mathrm{CH}_{2}+\mathrm{M} \rightleftharpoons \mathrm{C}+\mathrm{H}_{2}+\mathrm{M}$ & $1.30 \times 10^{14}$ & 0.0 & 29700.0 & 26 \\
\hline 31 & $\mathrm{CH}_{2}+\mathrm{M} \rightleftharpoons \mathrm{CH}+\mathrm{H}+\mathrm{M}$ & $4.00 \times 10^{15}$ & 0.0 & 41800.0 & 26 \\
\hline 32 & $\mathrm{CH}_{2}+\mathrm{N} \rightleftharpoons \mathrm{HCN}+\mathrm{H}$ & $5.00 \times 10^{13}$ & 0.0 & 0.0 & 27 \\
\hline 33 & $\mathrm{CH}_{2}+\mathrm{N}_{2} \rightleftharpoons \mathrm{HCN}+\mathrm{NH}$ & $4.82 \times 10^{12}$ & 0.0 & 18000.0 & 31 \\
\hline 34 & $\mathrm{CH}_{2}+\mathrm{NO} \rightleftharpoons \mathrm{OH}+\mathrm{HCN}$ & $2.90 \times 10^{14}$ & -0.69 & 382.70 & 18 \\
\hline 35 & $\mathrm{CH}_{3}+\mathrm{C} \rightleftharpoons \mathrm{C}_{2} \mathrm{H}_{2}+\mathrm{H}$ & $5.00 \times 10^{13}$ & 0.0 & 0.0 & 26 \\
\hline 36 & $\mathrm{CH}_{3}+\mathrm{H} \rightleftharpoons \mathrm{CH}_{2}+\mathrm{H}_{2}$ & $6.03 \times 10^{13}$ & 0.0 & 7600.0 & 30 \\
\hline 37 & $\mathrm{CH}_{3}+\mathrm{HCO} \rightleftharpoons \mathrm{CH}_{4}+\mathrm{CO}$ & $1.21 \times 10^{14}$ & 0.0 & 0.0 & 32 \\
\hline
\end{tabular}

Table 1: Reactions considered for the atmospheric ablating PICA chemistry model 
Table 1 - continued from previous page

\begin{tabular}{|c|c|}
\hline$\#$ & Reaction \\
\hline 38 & $\mathrm{CH}_{3}+\mathrm{M} \rightleftharpoons \mathrm{CH}+\mathrm{H}_{2}+\mathrm{M}$ \\
\hline 39 & $\mathrm{CH}_{3}+\mathrm{M} \rightleftharpoons \mathrm{CH}_{2}+\mathrm{H}+\mathrm{M}$ \\
\hline 40 & $\mathrm{CH}_{3}+\mathrm{N} \rightleftharpoons \mathrm{HCN}+\mathrm{H}+\mathrm{H}$ \\
\hline 41 & $\mathrm{CH}_{3}+\mathrm{N} \rightleftharpoons \mathrm{HCN}+\mathrm{H}_{2}$ \\
\hline 42 & $\mathrm{CH}_{3}+\mathrm{NO} \rightleftharpoons \mathrm{HCN}+\mathrm{H}_{2} \mathrm{O}$ \\
\hline 43 & $\mathrm{CH}_{4}+\mathrm{M} \rightleftharpoons \mathrm{CH}_{3}+\mathrm{H}+\mathrm{M}$ \\
\hline 44 & $\mathrm{CN}+\mathrm{C} \rightleftharpoons \mathrm{C}_{2}+\mathrm{N}$ \\
\hline 45 & $\mathrm{CN}+\mathrm{CO} \rightleftharpoons \mathrm{C}+\mathrm{NCO}$ \\
\hline 46 & $\mathrm{CN}+\mathrm{CO}_{2} \rightleftharpoons \mathrm{CO}+\mathrm{NCO}$ \\
\hline 47 & $\mathrm{CN}+\mathrm{H}_{2} \rightleftharpoons \mathrm{HCN}+\mathrm{H}$ \\
\hline 48 & $\mathrm{CN}+\mathrm{H}_{2} \mathrm{O} \rightleftharpoons \mathrm{HCN}+\mathrm{OH}$ \\
\hline 49 & $\mathrm{CN}+\mathrm{M} \rightleftharpoons \mathrm{C}+\mathrm{N}+\mathrm{M}$ \\
\hline 50 & $\mathrm{CN}+\mathrm{NO} \rightleftharpoons \mathrm{N}+\mathrm{NCO}$ \\
\hline 51 & $\mathrm{CN}+\mathrm{O} \rightleftharpoons \mathrm{CO}+\mathrm{N}$ \\
\hline 52 & $\mathrm{CN}+\mathrm{O}_{2} \rightleftharpoons \mathrm{O}+\mathrm{NCO}$ \\
\hline 53 & $\mathrm{CN}+\mathrm{OH} \rightleftharpoons \mathrm{NCO}+\mathrm{H}$ \\
\hline 54 & $\mathrm{CN}^{+}+\mathrm{N} \rightleftharpoons \mathrm{CN}+\mathrm{N}^{+}$ \\
\hline 55 & $\mathrm{C}+\mathrm{N} \rightleftharpoons \mathrm{CN}^{+}+\mathrm{e}$ \\
\hline 56 & $\mathrm{CO}+\mathrm{C}^{+} \rightleftharpoons \mathrm{CO}^{+}+\mathrm{C}$ \\
\hline 57 & $\mathrm{CO}+\mathrm{M} \rightleftharpoons \mathrm{O}+\mathrm{C}+\mathrm{M}$ \\
\hline 58 & $\mathrm{CO}_{2}+\mathrm{M} \rightleftharpoons \mathrm{O}+\mathrm{CO}+\mathrm{M}$ \\
\hline 59 & $\mathrm{CO}_{2}+\mathrm{O} \rightleftharpoons \mathrm{CO}+\mathrm{O}_{2}$ \\
\hline 60 & $\mathrm{H}+\mathrm{C}_{2} \mathrm{H}_{2} \rightleftharpoons \mathrm{C}_{2} \mathrm{H}+\mathrm{H}_{2}$ \\
\hline 61 & $\mathrm{H}+\mathrm{e} \rightleftharpoons \mathrm{H}^{+}+\mathrm{e}+\mathrm{e}$ \\
\hline 62 & $\mathrm{H}+\mathrm{CH}_{4} \rightleftharpoons \mathrm{CH}_{3}+\mathrm{H}_{2}$ \\
\hline 63 & $\mathrm{H}+\mathrm{H}_{2} \mathrm{O}_{2} \rightleftharpoons \mathrm{HO}_{2}+\mathrm{H}_{2}$ \\
\hline 64 & $\mathrm{H}+\mathrm{H}_{2} \mathrm{O}_{2} \rightleftharpoons \mathrm{OH}+\mathrm{H}_{2} \mathrm{O}$ \\
\hline 65 & $\mathrm{H}+\mathrm{HCO} \rightleftharpoons \mathrm{H}_{2}+\mathrm{CO}$ \\
\hline 66 & $\mathrm{H}+\mathrm{HO}_{2} \rightleftharpoons 2 \mathrm{OH}$ \\
\hline 67 & $\mathrm{H}+\mathrm{HO}_{2} \rightleftharpoons \mathrm{O}+\mathrm{H}_{2} \mathrm{O}$ \\
\hline 68 & $\mathrm{H}+\mathrm{HO}_{2} \rightleftharpoons \mathrm{O}_{2}+\mathrm{H}_{2}$ \\
\hline 69 & $\mathrm{H}+\mathrm{N}_{2} \rightleftharpoons \mathrm{NH}+\mathrm{N}$ \\
\hline 70 & $\mathrm{H}+\mathrm{NO}+\mathrm{M} \rightleftharpoons \mathrm{HNO}+\mathrm{M}$ \\
\hline
\end{tabular}

$\mathrm{H}+\mathrm{O}_{2}+\mathrm{N}_{2} \rightleftharpoons \mathrm{HO}_{2}+\mathrm{N}_{2}$

$\mathrm{H}+\mathrm{O}_{2}+\mathrm{N}_{2}^{+} \rightleftharpoons \mathrm{HO}_{2}+\mathrm{N}_{2}^{+}$

$\mathrm{H}+2 \mathrm{O}_{2} \rightleftharpoons \mathrm{HO}_{2}+\mathrm{O}_{2}$

$\mathrm{H}+2 \mathrm{O}_{2}^{+} \rightleftharpoons \mathrm{HO}_{2}+\mathrm{O}_{2}^{+}$

$\mathrm{H}+\mathrm{O}_{2}+\mathrm{H}_{2} \mathrm{O} \rightleftharpoons \mathrm{HO}_{2}+\mathrm{H}_{2} \mathrm{O}$

$$
\mathrm{H}+\mathrm{O}_{2} \rightleftharpoons \mathrm{O}+\mathrm{OH}
$$

$\mathrm{H}+\mathrm{OH}+\mathrm{M} \rightleftharpoons \mathrm{H}_{2} \mathrm{O}+\mathrm{M}$

A $[\mathrm{mol}, \mathrm{cm}, \mathrm{s}]$

$5.00 \times 10^{15}$

$1.02 \times 10^{16}$

$7.00 \times 10^{13}$

$3.70 \times 10^{12}$

$9.60 \times 10^{13}$

$4.70 \times 10^{47}$

$5.00 \times 10^{13}$

$1.50 \times 10^{16}$

$4.00 \times 10^{14}$

$2.95 \times 10^{5}$

$8.00 \times 10^{12}$

$2.53 \times 10^{14}$

$2.00 \times 10^{13}$

$2.41 \times 10^{14}$

$1.05 \times 10^{13}$

$4.00 \times 10^{13}$

$9.80 \times 10^{12}$

$1.00 \times 10^{15}$

$1.0 \times 10^{13}$

$2.30 \times 10^{19}$

$\mathrm{C}, \mathrm{C}^{+}$enhanced by 1.50

$\mathrm{N}, \mathrm{N}^{+}$enhanced by 1.50

$\mathrm{O}, \mathrm{O}^{+}$enhanced by 1.50

$\mathrm{H}, \mathrm{H}^{+}$enhanced by 1.50

$$
\begin{aligned}
& 3.50 \times 10^{14} \\
& 2.10 \times 10^{13} \\
& 6.62 \times 10^{13} \\
& 2.2 \times 10^{22} \\
& 1.32 \times 10^{4} \\
& 1.21 \times 10^{7} \\
& 1.00 \times 10^{13} \\
& 7.34 \times 10^{13} \\
& 8.40 \times 10^{13} \\
& 3.97 \times 10^{12} \\
& 4.48 \times 10^{13} \\
& 1.84 \times 10^{13} \\
& 4.48 \times 10^{19}
\end{aligned}
$$

$\mathrm{H}_{2}$ enhanced by 2.00

$\mathrm{H}_{2} \mathrm{O}$ enhanced by 6.00

$\mathrm{CH}_{4}$ enhanced by 2.00

$\mathrm{CO}, \mathrm{CO}^{+}$enhanced by 1.50

$\mathrm{CO}_{2}$ enhanced by 2.00

$$
2.80 \times 10^{18}
$$

0.0

18

$\mathrm{O}_{2}, \mathrm{O}_{2}^{+}$enhanced by 0.00

$\mathrm{H}_{2} \mathrm{O}$ enhanced by 0.00

$\mathrm{CO}, \mathrm{CO}^{+}$enhanced by 0.75

$\mathrm{CO}_{2}$ enhanced by 1.50

$\mathrm{N}_{2}, \mathrm{~N}_{2}^{+}$enhanced by 0.00

$\begin{array}{lccc}2.60 \times 10^{19} & -1.2 & 0.0 & 18 \\ 2.60 \times 10^{19} & -1.2 & 0.0 & 18 \\ 2.08 \times 10^{19} & -1.2 & 0.0 & 18 \\ 2.08 \times 10^{19} & -1.2 & 0.0 & 18 \\ 1.13 \times 10^{19} & -0.8 & 0.0 & 18 \\ 2.65 \times 10^{16} & -0.7 & 8581.1 & 18 \\ 2.20 \times 10^{22} & -2.0 & 0.0 & 18\end{array}$

$\mathrm{H}_{2}$ enhanced by 0.73

$\mathrm{H}_{2} \mathrm{O}$ enhanced by 3.65

$\mathrm{CH}_{4}$ enhanced by 2.00

$\begin{array}{cccc}2.23 \times 10^{14} & 0.0 & 48350.0 & 22 \\ 3.57 \times 10^{26} & -2.6 & 62845.0 & 37 \\ 3.91 \times 10^{9} & 1.6 & 13394.6 & 18 \\ 2.03 \times 10^{4} & 2.6 & 2507.7 & 18 \\ 5.07 \times 10^{3} & 2.6 & 2507.7 & 18 \\ 1.87 \times 10^{17} & -1.0 & 8560.5 & 18\end{array}$

Continued on next page 
Table $1-$ continued from previous page

\begin{tabular}{|c|c|}
\hline$\#$ & Reaction \\
\hline 85 & $\mathrm{HCO}+\mathrm{O}_{2} \rightleftharpoons \mathrm{HO}_{2}+\mathrm{CO}$ \\
\hline 86 & $\mathrm{HNO}+\mathrm{H} \rightleftharpoons \mathrm{H}_{2}+\mathrm{NO}$ \\
\hline 87 & $\mathrm{HNO}+\mathrm{O} \rightleftharpoons \mathrm{NO}+\mathrm{OH}$ \\
\hline 88 & $\mathrm{HNO}+\mathrm{O}_{2} \rightleftharpoons \mathrm{HO}_{2}+\mathrm{NO}$ \\
\hline 89 & $\mathrm{HNO}+\mathrm{OH} \rightleftharpoons \mathrm{NO}+\mathrm{H}_{2} \mathrm{O}$ \\
\hline 90 & $\mathrm{HO}_{2}+\mathrm{CO} \rightleftharpoons \mathrm{OH}+\mathrm{CO}_{2}$ \\
\hline 91 & $\mathrm{~N}+\mathrm{CO}_{2} \rightleftharpoons \mathrm{NO}+\mathrm{CO}$ \\
\hline 92 & $\mathrm{~N}+\mathrm{e} \rightleftharpoons \mathrm{N}^{+}+\mathrm{e}-+\mathrm{e}$ \\
\hline 93 & $\mathrm{~N}+\mathrm{H}_{2} \rightleftharpoons \mathrm{NH}+\mathrm{H}$ \\
\hline 94 & $\mathrm{~N}+\mathrm{N} \rightleftharpoons \mathrm{N}_{2}^{+}+\mathrm{e}$ \\
\hline 95 & $\mathrm{~N}+\mathrm{OH} \rightleftharpoons \mathrm{NO}+\mathrm{H}$ \\
\hline 96 & $\mathrm{~N}^{+}+\mathrm{N}_{2} \rightleftharpoons \mathrm{N}_{2}^{+}+\mathrm{N}$ \\
\hline 97 & $\mathrm{~N}_{2}+\mathrm{M} \rightleftharpoons \mathrm{N}+\mathrm{N}+\mathrm{M}$ \\
\hline
\end{tabular}

$\begin{array}{lc}98 & \mathrm{~N}_{2}+\mathrm{e} \rightleftharpoons \mathrm{N}+\mathrm{N}+\mathrm{e} \\ 99 & \mathrm{~N}_{2}+\mathrm{O} \rightleftharpoons \mathrm{N}+\mathrm{NO} \\ 100 & \mathrm{~N}_{2}+\mathrm{O}_{2}+\rightleftharpoons \mathrm{N}_{2}+\mathrm{O}_{2} \\ 101 & \mathrm{NCO}+\mathrm{H} \rightleftharpoons \mathrm{NH}+\mathrm{CO} \\ 102 & \mathrm{NCO}+\mathrm{M} \rightleftharpoons \mathrm{N}+\mathrm{CO}+\mathrm{M} \\ 103 & \mathrm{NCO}+\mathrm{N} \rightleftharpoons \mathrm{N}_{2}+\mathrm{CO} \\ 104 & \mathrm{NCO}+\mathrm{NO} \rightleftharpoons \mathrm{N}_{2}+\mathrm{CO}_{2} \\ 105 & \mathrm{NCO}+\mathrm{O} \rightleftharpoons \mathrm{NO}+\mathrm{CO} \\ 106 & \mathrm{NCO}+\mathrm{O}_{2} \rightleftharpoons \mathrm{NO}+\mathrm{CO}_{2} \\ 107 & \mathrm{NCO}+\mathrm{OH} \rightleftharpoons \mathrm{NO}+\mathrm{H}+\mathrm{CO} \\ 108 & \mathrm{NH}+\mathrm{CO}_{2} \rightleftharpoons \mathrm{HNO}+\mathrm{CO} \\ 109 & \mathrm{NH}+\mathrm{H}_{2} \mathrm{O} \rightleftharpoons \mathrm{HNO}+\mathrm{H}_{2} \\ 110 & \mathrm{NH}+\mathrm{M} \rightleftharpoons \mathrm{N}+\mathrm{H}+\mathrm{M} \\ 111 & \mathrm{NH}+\mathrm{NO} \rightleftharpoons \mathrm{N}+\mathrm{OH} \\ 112 & \mathrm{NH}+\mathrm{O} \rightleftharpoons \mathrm{NO}+\mathrm{H} \\ 113 & \mathrm{NH}+\mathrm{O}_{2} \rightleftharpoons \mathrm{HNO}+\mathrm{O} \\ 114 & \mathrm{NH}+\mathrm{O}_{2} \rightleftharpoons \mathrm{NO}+\mathrm{OH} \\ 115 & \mathrm{NH}+\mathrm{OH} \rightleftharpoons \mathrm{HNO}+\mathrm{H} \\ 116 & \mathrm{NH}+\mathrm{OH} \rightleftharpoons \mathrm{N}+\mathrm{H} \mathrm{H}_{2} \mathrm{O} \\ 117 & \mathrm{NO}+\mathrm{M} \rightleftharpoons \mathrm{N}+\mathrm{O}+\mathrm{M}\end{array}$

$\mathrm{A}[\mathrm{mol}, \mathrm{cm}, \mathrm{s}]$
$\mathrm{H}_{2}$ enhanced by
$\mathrm{H}_{2} \mathrm{O}$ enhanced by
$\mathrm{CH}_{4}$ enhanced by
$\mathrm{CO}, \mathrm{CO}^{+}$enhanced
$\mathrm{CO}_{2}$ enhanced by
$1.34 \times 10^{13}$
$9.00 \times 10^{11}$
$2.50 \times 10^{13}$
$1.00 \times 10^{13}$
$1.30 \times 10^{7}$
$1.50 \times 10^{14}$
$3.00 \times 10^{12}$
$2.5 \times 10^{34}$
$1.60 \times 10^{14}$
$2.0 \times 10^{13}$
$3.36 \times 10^{13}$
$1.0 \times 10^{12}$
$7.00 \times 10^{21}$

$\mathrm{N}, \mathrm{N}^{+}$enhanced by 4.28

$\mathrm{O}, \mathrm{O}^{+}$enhanced by 4.28

$\mathrm{C}, \mathrm{C}^{+}$enhanced by 4.28

$\mathrm{H}, \mathrm{H}^{+}$enhanced by 4.28$$
7.00 \times 10^{24}
$$$$
6.40 \times 10^{17}
$$$$
9.9 \times 10^{12}
$$$$
5.40 \times 10^{13}
$$$$
6.30 \times 10^{16}
$$$$
2.00 \times 10^{13}
$$$$
3.80 \times 10^{18}
$$$$
2.35 \times 10^{13}
$$$$
2.00 \times 10^{12}
$$$$
2.50 \times 10^{12}
$$$$
1.00 \times 10^{13}
$$$$
2.00 \times 10^{13}
$$$$
1.80 \times 10^{14}
$$$$
2.16 \times 10^{13}
$$$$
4.00 \times 10^{13}
$$$$
4.61 \times 10^{5}
$$$$
1.28 \times 10^{6}
$$$$
2.00 \times 10^{13}
$$$$
2.00 \times 10^{9}
$$$$
5.00 \times 10^{15}
$$

$\mathrm{C}, \mathrm{C}^{+}$enhanced by 20.0

$\mathrm{N}, \mathrm{N}^{+}$enhanced by 20.0

$\mathrm{O}, \mathrm{O}^{+}$enhanced by 20.0

$\mathrm{H}, \mathrm{H}^{+}$enhanced by 20.0

$\begin{array}{cccc}8.40 \times 10^{12} & 0.0 & 19450.0 & 8 \\ 1.0 \times 10^{13} & 0.00 & 23200.0 & 33 \\ 7.20 \times 10^{13} & 0.0 & 35500.0 & 38 \\ 3.4 \times 10^{13} & 0.00 & 12800.0 & 33 \\ 1.0 \times 10^{12} & 0.50 & 77200 . & 38 \\ 7.2 \times 10^{12} & 0.29 & 48600.0 & 33 \\ 2.4 \times 10^{13} & 0.41 & 32600 . & 38 \\ 5.00 \times 10^{13} & 0.0 & 0.0 & 18 \\ 6.94 \times 10^{6} & 2.0 & 956.8 & 18 \\ 4.60 \times 10^{19} & -1.4 & 14577.9 & 18 \\ 5.70 \times 10^{13} & 0.0 & 0.0 & 18 \\ 8.00 \times 10^{13} & 0.0 & 0.0 & 18 \\ 3.37 \times 10^{13} & 0.0 & 0.0 & 18 \\ 1.02 \times 10^{9} & 1.5 & 4330.6 & 18 \\ 3.9 \times 10^{33} & -3.78 & 158500.0 & 33 \\ 5.00 \times 10^{17} & -1.0 & 0.0 & 18\end{array}$

$\mathrm{H}_{2}$ enhanced by 2.00

$\mathrm{H}_{2} \mathrm{O}$ enhanced by 6.00

$\begin{array}{ccc}0.0 & 201.4 & 18 \\ 0.7 & 332.4 & 18 \\ 0.0 & 0.0 & 18 \\ 0.0 & 6546.2 & 18 \\ 1.9 & -478.4 & 18 \\ 0.0 & 11883.9 & 18 \\ 0.0 & 5690.2 & 18 \\ -3.82 & 168600.0 & 8 \\ 0.0 & 12650.0 & 34 \\ 0.00 & 67500 . & 38 \\ 0.0 & 193.9 & 18 \\ 0.50 & 12200.0 & 38 \\ -1.6 & 113200.0 & 8\end{array}$

$\begin{array}{ccc}-1.6 & 113200.0 & 8 \\ -1.0 & 38370.0 & 8 \\ 0.00 & 40700 . & 38 \\ 0.0 & 0.0 & 18 \\ -0.5 & 24000.0 & 33 \\ 0.0 & 0.0 & 18 \\ -2.0 & 402.8 & 18 \\ 0.0 & 0.0 & 18 \\ 0.00 & 10071.11 & 18 \\ 0.0 & 0.0 & 18 \\ 0.0 & 7226.0 & 18 \\ 0.0 & 6974.2 & 18 \\ 0.0 & 37600.0 & \\ -0.2 & 0.0 & 18 \\ 0.0 & 0.0 & 18 \\ 2.0 & 3273.1 & 18 \\ 1.5 & 50.4 & 18 \\ 0.0 & 0.0 & 18 \\ 1.2 & 0.0 & 18 \\ 0.0 & 75500.0 & 33\end{array}$

Continued on next page

6 of 18 
Table 1 - continued from previous page

\begin{tabular}{|c|c|c|c|c|c|}
\hline$\#$ & Reaction & $\mathrm{A}[\mathrm{mol}, \mathrm{cm}, \mathrm{s}]$ & $\mathrm{n}$ & $T_{a}[\mathrm{~K}]$ & References \\
\hline & & $\mathrm{CH}_{4}$ enhanced by 2.00 & & & \\
\hline & & $\mathrm{CO}, \mathrm{CO}^{+}$enhanced by 1.50 & & & \\
\hline & & $\mathrm{CO}_{2}$ enhanced by 2.00 & & & \\
\hline 134 & $\mathrm{O}+\mathrm{H}_{2} \rightleftharpoons \mathrm{H}+\mathrm{OH}$ & $3.87 \times 10^{4}$ & 2.7 & 3152.3 & 18 \\
\hline 135 & $\mathrm{O}+\mathrm{H}_{2} \mathrm{O}_{2} \rightleftharpoons \mathrm{OH}+\mathrm{HO}_{2}$ & $9.63 \times 10^{6}$ & 2.0 & 2014.2 & 18 \\
\hline 136 & $\mathrm{O}+\mathrm{HCO} \rightleftharpoons \mathrm{H}+\mathrm{CO}_{2}$ & $3.00 \times 10^{13}$ & 0.0 & 0.0 & 18 \\
\hline 137 & $\mathrm{O}+\mathrm{HCO} \rightleftharpoons \mathrm{OH}+\mathrm{CO}$ & $3.00 \times 10^{13}$ & 0.0 & 0.0 & 18 \\
\hline 138 & $\mathrm{O}+\mathrm{HO}_{2} \rightleftharpoons \mathrm{OH}+\mathrm{O}_{2}$ & $2.00 \times 10^{13}$ & 0.0 & 0.0 & 18 \\
\hline 139 & $\mathrm{O}+\mathrm{N} \rightleftharpoons \mathrm{NO}^{+}+\mathrm{e}$ & $5.30 \times 10^{12}$ & 0.0 & 31900.0 & 33 \\
\hline 140 & $\mathrm{O}+\mathrm{O} \rightleftharpoons \mathrm{O}_{2}^{+}+\mathrm{e}$ & $1.1 \times 10^{13}$ & 0.00 & 80600. & 38 \\
\hline 141 & $\mathrm{O}^{+}+\mathrm{NO} \rightleftharpoons \mathrm{N}^{+}+\mathrm{O}_{2}$ & $1.4 \times 10^{5}$ & 1.90 & 15300. & 38 \\
\hline 142 & $\mathrm{O}^{+}+\mathrm{N}_{2} \rightleftharpoons \mathrm{N}_{2}^{+}+\mathrm{O}$ & $9.1 \times 10^{11}$ & 0.36 & 22800 . & 38 \\
\hline 143 & $\mathrm{O}_{2}^{+}+\mathrm{N} \rightleftharpoons \mathrm{N}^{+}+\mathrm{O}_{2}$ & $8.70 \times 10^{13}$ & 0.1 & 28600.0 & 38 \\
\hline 144 & $\mathrm{O}_{2}^{+}+\mathrm{O} \rightleftharpoons \mathrm{O}_{2}+\mathrm{O}^{+}$ & $4.0 \times 10^{12}$ & -0.09 & 18000.0 & 33 \\
\hline 145 & $\mathrm{O}_{2}+\mathrm{C}^{+} \rightleftharpoons \mathrm{O}_{2}^{+}+\mathrm{C}$ & $1.0 \times 10^{13}$ & 0.00 & 9400.0 & 33 \\
\hline \multirow[t]{5}{*}{146} & $\mathrm{O}_{2}+\mathrm{M} \rightleftharpoons \mathrm{O}+\mathrm{O}+\mathrm{M}$ & $2.00 \times 10^{21}$ & -1.5 & 59500.0 & 8 \\
\hline & & $\mathrm{N}, \mathrm{N}^{+}$enhanced by 5.00 & & & \\
\hline & & $\mathrm{O}, \mathrm{O}^{+}$enhanced by 5.00 & & & \\
\hline & & $\mathrm{H}, \mathrm{H}^{+}$enhanced by 5.00 & & & \\
\hline & & $\mathrm{C}, \mathrm{C}^{+}$enhanced by 5.00 & & & \\
\hline 147 & $\mathrm{OH}+\mathrm{C} \rightleftharpoons \mathrm{H}+\mathrm{CO}$ & $5.00 \times 10^{13}$ & 0.0 & 0.0 & 18 \\
\hline 148 & $\mathrm{OH}+\mathrm{C}_{2} \mathrm{H}_{2} \rightleftharpoons \mathrm{CH}_{3}+\mathrm{CO}$ & $4.83 \times 10^{-4}$ & 4.0 & -1007.1 & 18 \\
\hline 149 & $\mathrm{OH}+\mathrm{CH} \rightleftharpoons \mathrm{H}+\mathrm{HCO}$ & $3.00 \times 10^{13}$ & 0.0 & 0.0 & 18 \\
\hline 150 & $\mathrm{OH}+\mathrm{CH}_{2} \rightleftharpoons \mathrm{CH}+\mathrm{H}_{2} \mathrm{O}$ & $1.13 \times 10^{7}$ & 2.0 & 1510.7 & 18 \\
\hline 151 & $\mathrm{OH}+\mathrm{CH}_{3} \rightleftharpoons \mathrm{CH}_{2}+\mathrm{H}_{2} \mathrm{O}$ & $5.60 \times 10^{7}$ & 1.6 & 2729.3 & 18 \\
\hline 152 & $\mathrm{OH}+\mathrm{CH}_{4} \rightleftharpoons \mathrm{CH}_{3}+\mathrm{H}_{2} \mathrm{O}$ & $1.37 \times 10^{6}$ & 2.2 & 1350.0 & 39 \\
\hline 153 & $\mathrm{OH}+\mathrm{CO} \rightleftharpoons \mathrm{H}+\mathrm{CO}_{2}$ & $4.76 \times 10^{7}$ & 1.2 & 35.2 & 18 \\
\hline 154 & $\mathrm{OH}+\mathrm{C}_{2} \mathrm{H}_{2} \rightleftharpoons \mathrm{C}_{2} \mathrm{H}+\mathrm{H}_{2} \mathrm{O}$ & $3.37 \times 10^{7}$ & 2.00 & 7049.8 & 18 \\
\hline 155 & $\mathrm{OH}+\mathrm{H}_{2} \rightleftharpoons \mathrm{H}+\mathrm{H}_{2} \mathrm{O}$ & $2.16 \times 10^{8}$ & 1.5 & 1727.2 & 18 \\
\hline 156 & $\mathrm{OH}+\mathrm{H}_{2} \mathrm{O}_{2} \rightleftharpoons \mathrm{HO}_{2}+\mathrm{H}_{2} \mathrm{O}$ & $2.00 \times 10^{12}$ & 0.0 & 215.0 & 18 \\
\hline 157 & $\mathrm{OH}+\mathrm{HCO} \rightleftharpoons \mathrm{H}_{2} \mathrm{O}+\mathrm{CO}$ & $5.00 \times 10^{13}$ & 0.0 & 0.0 & 18 \\
\hline 158 & $\mathrm{OH}+\mathrm{HO}_{2} \rightleftharpoons \mathrm{O}_{2}+\mathrm{H}_{2} \mathrm{O}$ & $1.45 \times 10^{13}$ & 0.0 & -251.8 & 18 \\
\hline
\end{tabular}

\section{B. Experimental comparisons}

In order to validate the model, multiple zero-dimensional simulations are performed using the CHEMKIN ${ }^{20}$ package, and compared to available experimental data. The experiments presented here are selected because they are typical of an ablative boundary layer during hypersonic re-entry. As can be seen in Fig. 2, the model fits the data remarkably well, which is expected since some of the presented experiments were employed to validate and even compute the kinetic rates used in the model. The discrepancy observed in some of the results, especially those presented in Fig. 2b), c) and f) is caused by the choice of a different reaction rate than the one obtained from the experimental data. As shown earlier, changing one kinetic rate can have a non trivial impact on the predicted concentration of the species over time. A detailed explanation of this is presented in Ref. 19. The results presented here, although not perfect, are considered to agree remarkably well with the experimental data.

\section{LeMANS: an unstructured three-dimensional Navier-Stokes solver for hypersonic nonequilibrium aerothermodynamics}

The hypersonic aerothermodynamic CFD code used to analyze the chemistry model is LeMANS, a finite volume Navier-Stokes solver currently being developed at The University of Michigan. ${ }^{43-46}$ The code assumes that the rotational and translational energy modes of all species can be described by their respective temperatures $T_{r}$ and $T$, and that the vibrational energy mode and electronic energy mode of all species, as well as the free electron kinetic energy, can be described by a single temperature, $T_{\text {vee }}{ }^{47}$ The viscous stresses are modeled assuming a Newtonian fluid, using Stokes' hypothesis, and the species mass diffusion fluxes are modeled using a modified version of Fick's law. Mixture transport properties are calculated using one of two models; the first uses Wilke's semi-empirical mixing rule with species viscosities calculated using Blottner's 


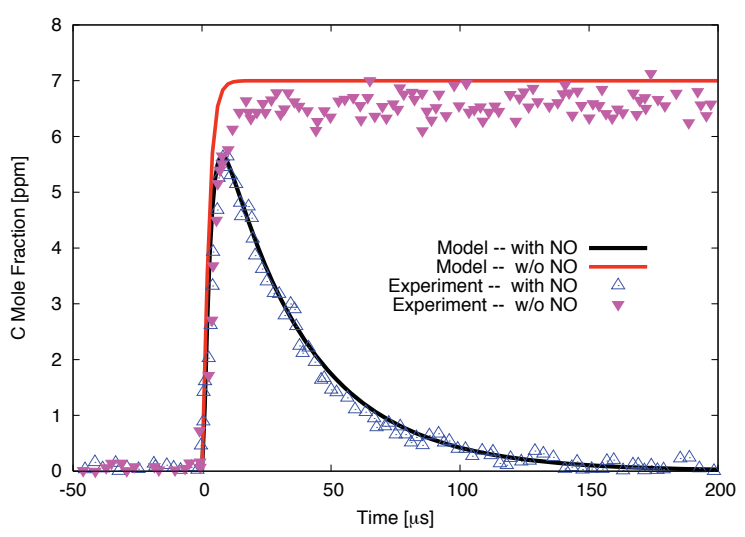

(a) Comparison to the experiment of Ref. 40: $T=2864 \mathrm{~K}$, $p=1.00 \mathrm{~atm}, 7 \mathrm{ppm}$ of $\mathrm{C}_{3} \mathrm{O}_{2}$ and $147 \mathrm{ppm}$ of $\mathrm{NO}$ in Argon

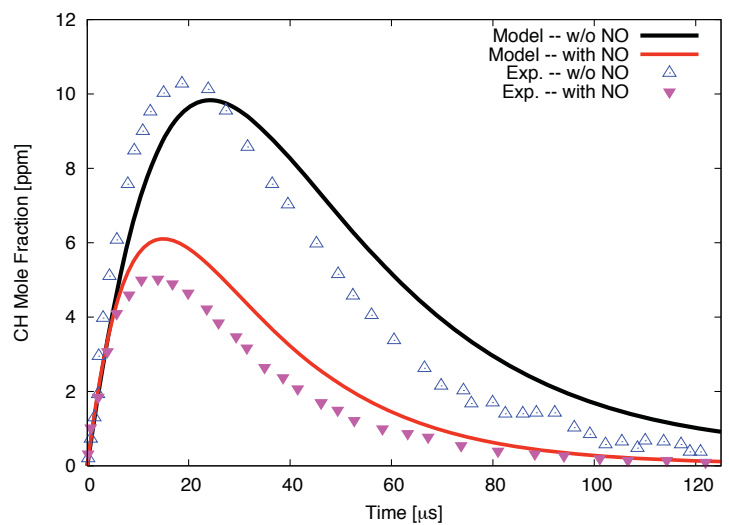

(c) Comparison to the experiment of Ref. 40: $T=3287$ $\mathrm{K}, p=0.67 \mathrm{~atm}, 20 \mathrm{ppm}$ of $\mathrm{C}_{2} \mathrm{H}_{6}$ and $300 \mathrm{ppm}$ of $\mathrm{NO}$ in Argon

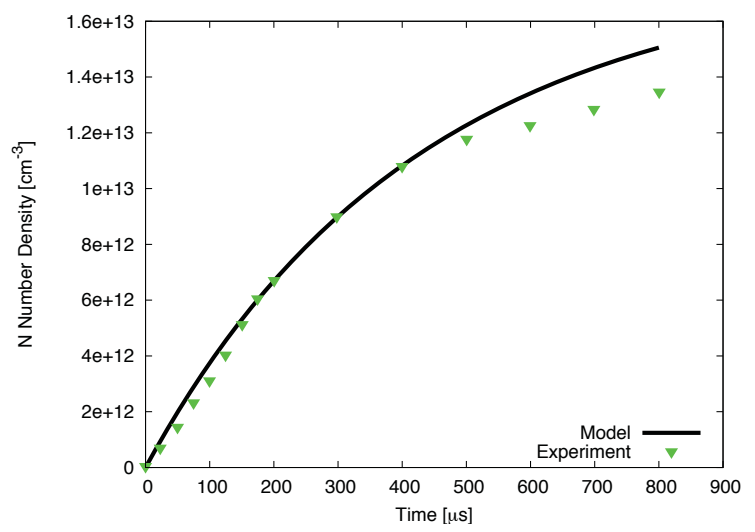

(e) Comparison to the experiment of Ref. 41: $T=5290 \mathrm{~K}$, $p=1.88 \mathrm{~atm}, 5 \mathrm{ppm}$ of $\mathrm{C}_{2} \mathrm{~N}_{2}$ in Argon

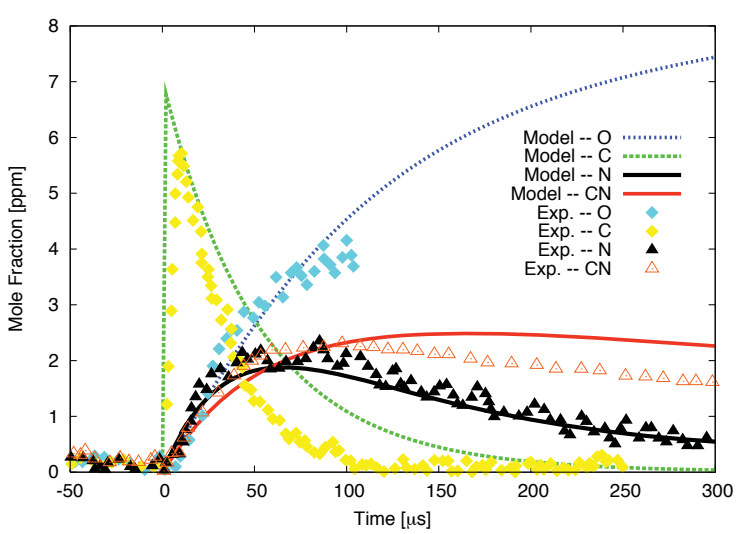

(b) Comparison to the experiment of Ref. 40: $T=3620$ $\mathrm{K}, p=0.80$ atm, $7 \mathrm{ppm}$ of $\mathrm{C}_{3} \mathrm{O}_{2}$ and $147 \mathrm{ppm}$ of $\mathrm{NO}$ in Argon

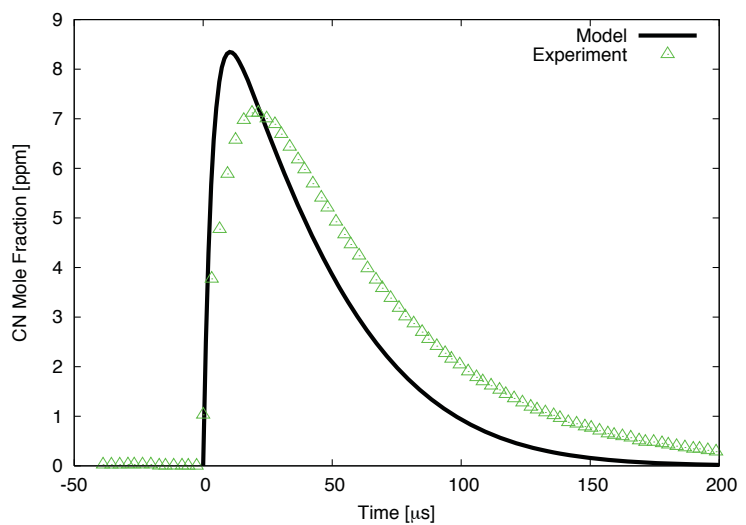

(d) Comparison to the experiment of Ref. 34: $T=3470$ $\mathrm{K}, p=0.835 \mathrm{~atm}, 5 \mathrm{ppm}$ of $\mathrm{C}_{2} \mathrm{~N}_{2}$ and $515 \mathrm{ppm}$ of $\mathrm{O}_{2}$ in Argon

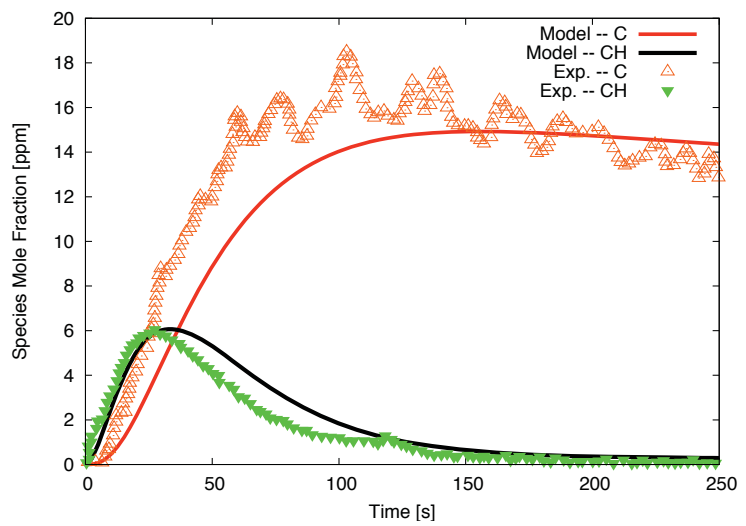

(f) Comparison to the experiment of Ref. $42: T=3100 \mathrm{~K}$, $p=0.89$ atm, $30 \mathrm{ppm}$ of $\mathrm{CH}_{4}$ in Argon

Figure 2. Evaluation of the chemistry model using experimental data 
model and species thermal conductivities determined using Eucken's relation, and the other uses Gupta's mixing rule with species viscosities and thermal conductivities calculated using non-coulombic/coulombic collision cross section data. Heat fluxes are modeled according to Fourier's law for all temperatures. Finally, the source terms of the species conservation equations are modeled using a standard finite-rate chemistry model for reacting air in conjunction with Park's two-temperature model to account for thermal nonequilibrium effects on the reaction rates.

Numerically, the code has the capability to handle meshes containing any mix of hexahedra, tetrahedra, prisms and pyramids in 3D, or triangles and quadrilaterals in 2D. Numerical fluxes between the cells are discretized using a modified Steger-Warming Flux Vector Splitting scheme, which has low dissipation and is appropriate to calculate boundary layers. A point or line implicit method is used to perform the time integration. The code has been extensively validated against experimental data, and has also been compared to other similar codes such as NASA Ames' DPLR ${ }^{14}$ and NASA Langley's LAURA. ${ }^{48}$

To account for the coupling between the flow field and the material response, the effects of ablation are added to the CFD code; therefore, a modification to the surface boundary condition is necessary. The physical values at the wall are obtained by solving the conservation of momentum:

$$
p_{n c}+\rho_{n c} v_{n c}^{2}=p_{w}\left(\rho_{w}, T_{w}\right)+\rho_{w} v_{w}^{2}
$$

as well as the species surface mass balance equation:

$$
\rho_{w} D_{w s} \frac{\partial Y_{w s}}{\partial \eta}=\dot{m}\left(Y_{g_{s}}-Y_{w s}\right)
$$

The surface energy balance equation does not need to be solved in the present anaysis as the wall temperature, blowing rates and blowing species are imposed and not calculated.

Once values are computed for the primitive variables, the conservative quantities in the ghost cells of the boundary are set such that the flux across the wall is the required blowing flux. This blowing boundary condition has been tested over a wide range of blowing rates, assuring the robustness of the implementation. Following the same methodology for the verification and validation of NASA Ames' DPLR code ${ }^{49}$ and NASA Langley's LAURA code, ${ }^{50}$ the blowing boundary of LeMANS has also been verified and validated..$^{7,51}$

\section{Test-case: Stardust re-entry vehicle}

\section{A. Problem description}

In order to evaluate and validate the model in LeMANS, the forebody of the Stardust return capsule is modeled during the first 10 seconds of its re-entry in the continuum regime. ${ }^{52}$ These re-entry points are chosen because they also correspond to the period where spectral emission data was measured by the Echelle instrumentation. The geometry and mesh are presented in Fig. 3 and the flow and surface parameters in Tables 2 and 3, respectively. Gibb's Free Energy is used to calculate the equilibrium constants needed for the backward reaction rates, and the transport properties are calculated from Lennard-Jones potentials, using CHEMKIN,${ }^{20}$ to produce individual species temperature dependant viscosity curve fits.

Table 2. Free stream conditions for the Echelle period of the trajectory of the Stardust re-entry vehicle

\begin{tabular}{cccccccc}
\hline $\begin{array}{c}\text { Time } \\
{[\mathrm{s}]}\end{array}$ & $\begin{array}{c}\text { Altitude } \\
{[\mathrm{km}]}\end{array}$ & $\begin{array}{c}U_{\infty} \\
{[\mathrm{km} / \mathrm{s}]}\end{array}$ & $\begin{array}{c}T_{\infty} \\
{[\mathrm{K}]}\end{array}$ & $\begin{array}{c}\rho_{\infty} \\
{\left[\mathrm{kg} / \mathrm{m}^{3}\right]}\end{array}$ & $Y_{N_{2}}$ & $Y_{O_{2}}$ & $\mathrm{Kn}$ \\
\hline 34 & 81.0 & 12.4 & 218. & $1.27 \times 10^{-4}$ & 0.763 & 0.237 & 0.00645 \\
36 & 78.5 & 12.3 & 218. & $1.87 \times 10^{-4}$ & 0.763 & 0.237 & 0.00432 \\
38 & 76.0 & 12.3 & 219. & $2.72 \times 10^{-4}$ & 0.763 & 0.237 & 0.00292 \\
40 & 73.5 & 12.2 & 220. & $3.92 \times 10^{-4}$ & 0.763 & 0.237 & 0.00203 \\
42 & 71.2 & 12.1 & 222. & $5.55 \times 10^{-4}$ & 0.763 & 0.237 & 0.00145 \\
44 & 68.9 & 11.9 & 224. & $7.72 \times 10^{-4}$ & 0.763 & 0.237 & 0.00105 \\
\hline \hline
\end{tabular}

The values obtained for the surface temperature and blowing rates are only valid at the stagnation point. Those two parameters are expected to be significantly lower elsewhere on the forebody of the vehicle. To 


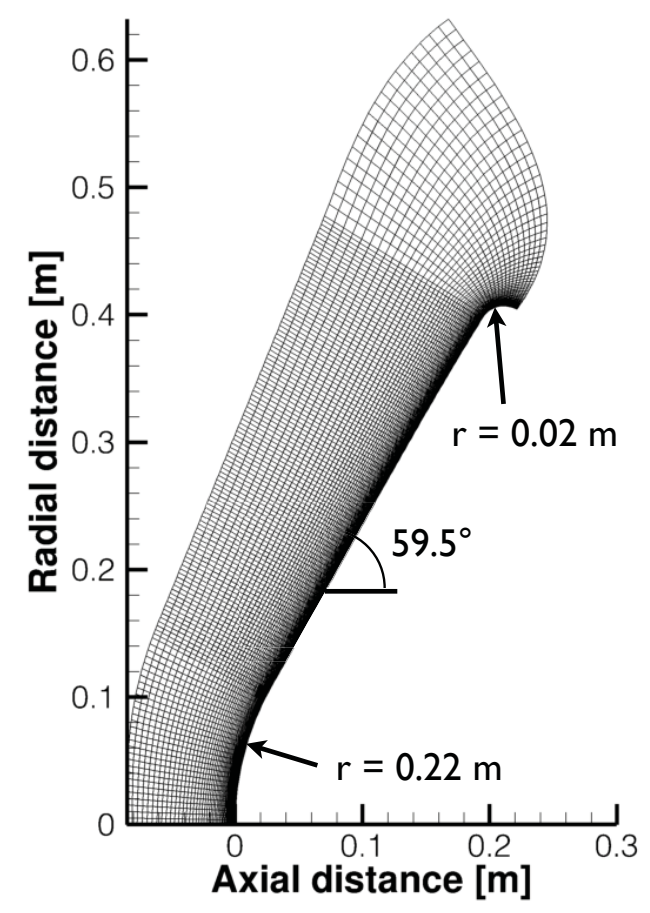

(a) Geometry and overall mesh

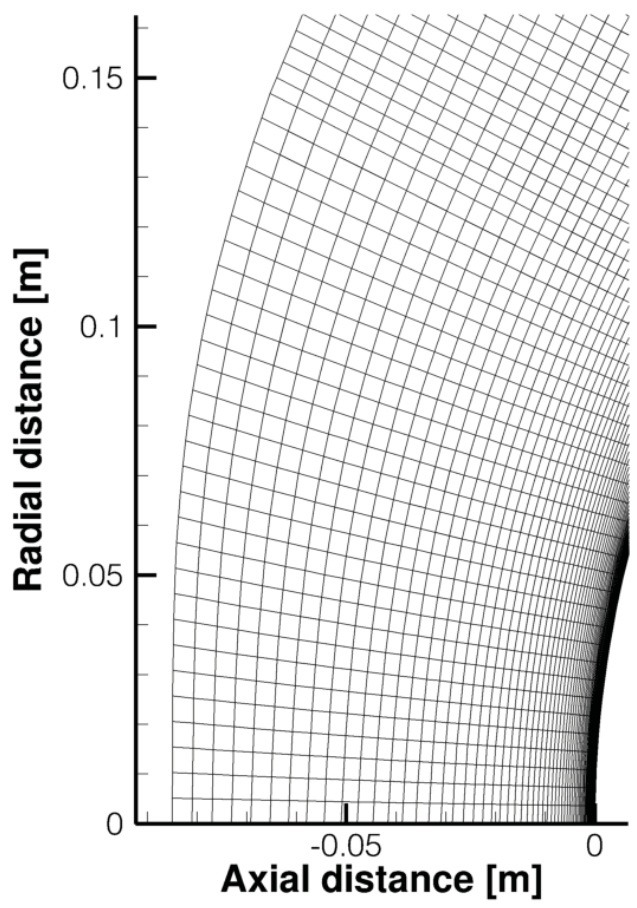

(b) Stagnation region mesh

Figure 3. Geometry and mesh of the Stardust re-entry capsule, used for the chemistry model comparison

Table 3. Wall condition at the stagnation point for the Echelle period of the trajectory of the Stardust re-entry vehicle

\begin{tabular}{ccccccc}
\hline \hline $\begin{array}{c}\text { Time } \\
{[\mathrm{s}]}\end{array}$ & $\begin{array}{c}T_{w} \\
{[\mathrm{~K}]}\end{array}$ & $\begin{array}{c}\dot{m}_{w} \\
{\left[\mathrm{~kg} / \mathrm{m}^{2} / \mathrm{s}\right]}\end{array}$ & $Y_{N_{2}}$ & $Y_{C O}$ & $Y_{H_{2}}$ & $Y_{\mathrm{H}_{2} O}$ \\
\hline 34 & 2890. & 0.0214 & $6.16 \times 10^{-1}$ & $2.32 \times 10^{-1}$ & $1.52 \times 10^{-2}$ & $6.85 \times 10^{-2}$ \\
36 & 2970. & 0.0264 & $6.14 \times 10^{-1}$ & $2.34 \times 10^{-1}$ & $1.52 \times 10^{-2}$ & $7.32 \times 10^{-2}$ \\
38 & 3070. & 0.0299 & $6.49 \times 10^{-1}$ & $1.75 \times 10^{-1}$ & $1.08 \times 10^{-2}$ & $4.88 \times 10^{-2}$ \\
40 & 3150. & 0.0378 & $6.54 \times 10^{-1}$ & $1.65 \times 10^{-1}$ & $9.38 \times 10^{-3}$ & $5.41 \times 10^{-2}$ \\
42 & 3240. & 0.0453 & $6.75 \times 10^{-1}$ & $1.23 \times 10^{-1}$ & $5.76 \times 10^{-3}$ & $5.23 \times 10^{-2}$ \\
44 & 3310. & 0.0549 & $6.78 \times 10^{-1}$ & $1.25 \times 10^{-1}$ & $7.21 \times 10^{-3}$ & $3.08 \times 10^{-2}$ \\
\hline \hline & & & & & \\
\hline \hline Time & $Y_{O H}$ & $Y_{O}$ & $Y_{C O_{2}}$ & $Y_{N O}$ & $Y_{O_{2}}$ & $Y_{N}$ \\
{$[\mathrm{~s}]$} & & & & & & \\
\hline 34 & $1.94 \times 10^{-2}$ & $1.24 \times 10^{-2}$ & $2.72 \times 10^{-2}$ & $4.93 \times 10^{-3}$ & $5.05 \times 10^{-3}$ & 0.00 \\
36 & $1.72 \times 10^{-2}$ & $9.18 \times 10^{-3}$ & $2.94 \times 10^{-2}$ & $4.38 \times 10^{-3}$ & $4.02 \times 10^{-3}$ & 0.00 \\
38 & $3.21 \times 10^{-2}$ & $3.75 \times 10^{-2}$ & $1.92 \times 10^{-2}$ & $1.22 \times 10^{-3}$ & $1.52 \times 10^{-2}$ & 0.00 \\
40 & $3.19 \times 10^{-2}$ & $3.33 \times 10^{-2}$ & $2.30 \times 10^{-2}$ & $1.30 \times 10^{-2}$ & $1.72 \times 10^{-2}$ & 0.00 \\
42 & $3.30 \times 10^{-2}$ & $3.68 \times 10^{-2}$ & $2.70 \times 10^{-2}$ & $1.74 \times 10^{-2}$ & $2.99 \times 10^{-2}$ & 0.00 \\
44 & $3.71 \times 10^{-2}$ & $6.46 \times 10^{-2}$ & $1.22 \times 10^{-2}$ & $2.08 \times 10^{-2}$ & $2.44 \times 10^{-2}$ & $7.84 \times 10^{-5}$ \\
\hline \hline
\end{tabular}


reflect this, a temperature profile is obtained using a fully radiative equilibrium boundary condition at the wall, without ablation, for the first re-entry point. This method has been proven to give a good estimate of the surface conditions. ${ }^{52}$ The surface temperature profile is then normalized and re-applied for the ablating wall simulation, multiplied by the surface temperature at the stagnation point that is listed in Table 3 . For the blowing rate, a linear relation between the temperature is derived using the first two columns of Table 3 , and applied proportionally using the wall temperature profile. The values for those two parameters are presented in Fig. 4.

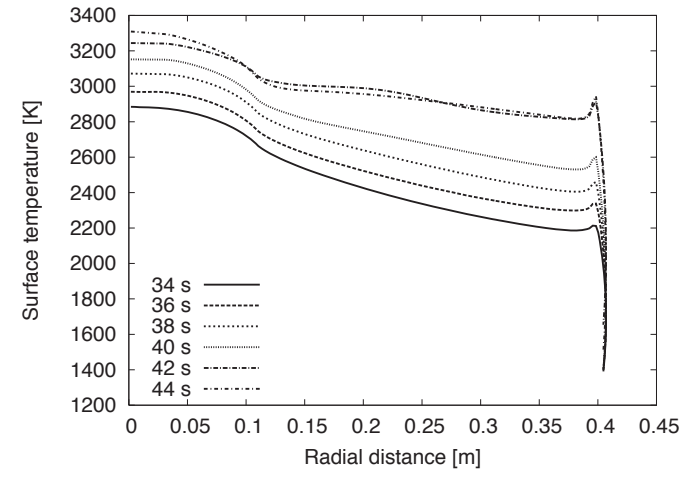

(a) Surface temperature

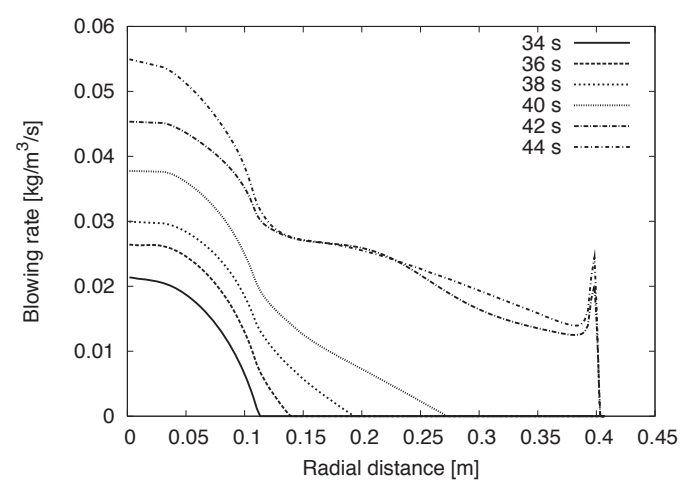

(b) Surface blowing rate

Figure 4. Calculated temperature and blowing rates for various re-entry points of the Stardust re-entry

\section{B. Results}

\section{Convective heat flux and Species concentrations}

The convective heat flux obtained with the ablative boundary condition, using the proposed chemistry model, is presented in Fig. 5 for the $34 \mathrm{~s}$ trajectory point. As expected, the heat flux is significantly reduced when compared to the radiative equilibrium heat flux, also presented on that figure. As can be seen, most of the heat reduction comes in the form of the translational-rotational conduction term. This is caused by two phenomena. First, the gradient of temperatures is slightly modified by the fact that the shock is pushed back by the blowing at the surface. Secondly, the composition of the boundary layer is not the same, which results in a modified total thermal conductivity. Past results ${ }^{12}$ tend to favor the latter phenomenon.

The species composition along the stagnation line is also presented in Fig. 6. It is interesting to note in Fig. 6b) that three of the blowing species $\left(\mathrm{CO}_{2}, \mathrm{H}_{2}\right.$ and $\left.\mathrm{H}_{2} \mathrm{O}\right)$ are destroyed almost immediately once they enter the flow, however, $\mathrm{CO}$ and $\mathrm{OH}$ remain in relatively high concentration. These two species are important since they are strong radiators. In Fig. 6c), it can be seen that two atomic species, $\mathrm{H}$ and C, are created in high concentration near the boundary, as are $\mathrm{CN}$ and $\mathrm{CN}^{+}$, which are quite important because of their radiative properties. It is also interesting to note, on that same figure, that the concentration of HNO remains high; this species was neglected in past models. ${ }^{8-10}$ Finally, Fig. 6d) presents the species which are not present in a significant concentration (species that had a number density less than the machine precision are not shown). Although it might be tempting to discard them, they remain important since they start to appear in greater concentration at other re-entry conditions (i.e. other altitudes). The same calculations were performed for all the trajectory points listed in Table 2, and although some differences are noticeable, the general conclusions remain the same. Results for the $38 \mathrm{~s}$ and $42 \mathrm{~s}$ trajectory ponts are presented in Figs. 7 and 8.

\section{Radiative heat flux}

Certain species present in the boundary layer are strong radiative emitters, and are expected to significantly contribute to the overall radiative heating to the surface. For the Stardust re-entry vehicle, the contribution 


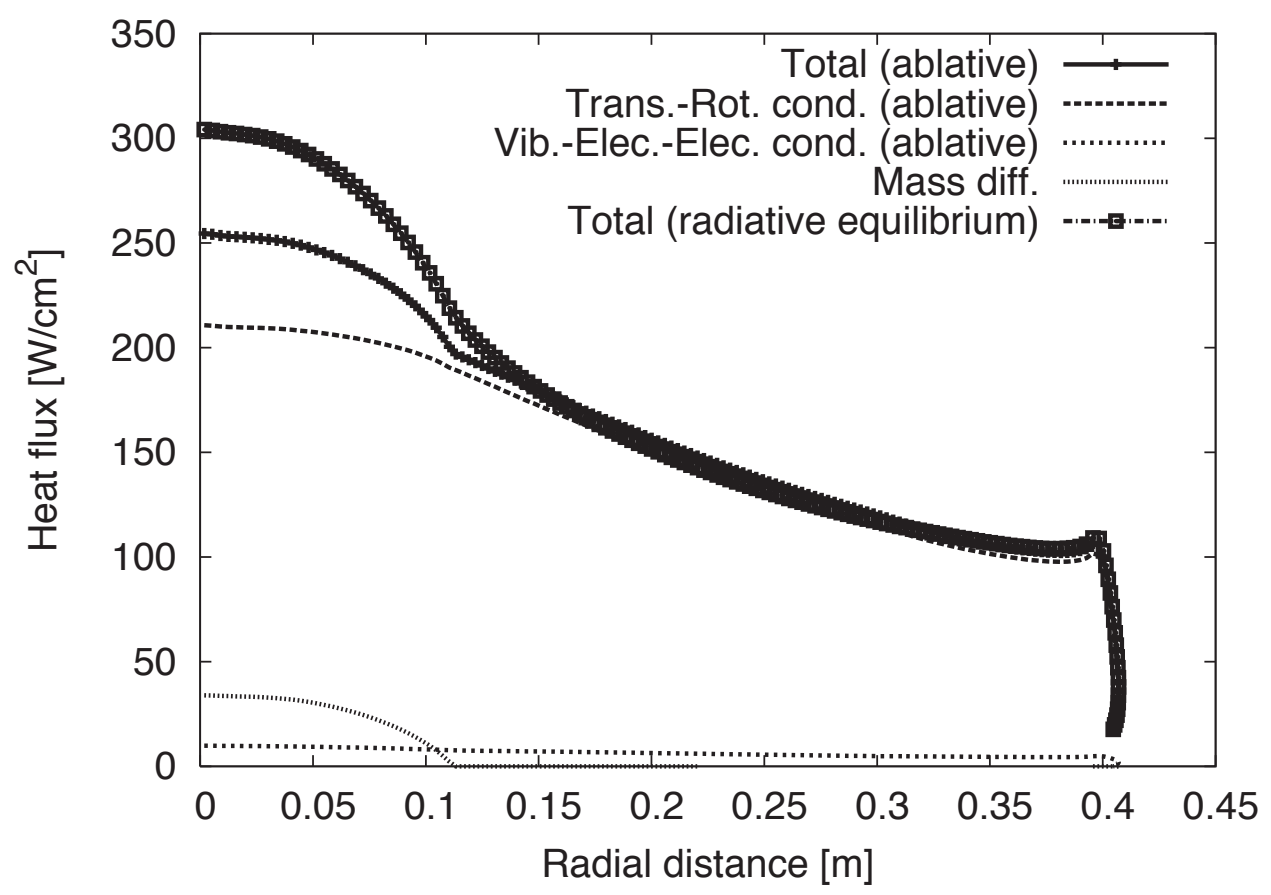

Figure 5. Heat fluxes at the surface of the Startdust re-entry vehicle at 34 seconds $(81 \mathrm{~km})$

of the radiative heat flux to the overall heat flux has been evaluated at approximately $10 \%$.

A radiative emission calculation is performed along the stagnation line of a converged flow field solution of the $42 \mathrm{~s}$ trajectory point, using the non-equilibrium radiation code NEQAIR (version 99d). ${ }^{13}$ Apart from the usual air species, radiative emission from the species listed in Table 4 is included.

Table 4. Emission mechanisms considered for the calculation of the radiative heat flux, excluding the air species

\begin{tabular}{cc}
\hline \hline Species & Mechanism \\
\hline $\mathrm{CN}$ & Violet $\left(\mathrm{B}^{1} \Sigma-\mathrm{X}^{1} \Sigma\right)$ \\
$\mathrm{CO}$ & $4+\left(\mathrm{A}^{1} \Pi-\mathrm{X}^{1} \Sigma\right)$ \\
$\mathrm{C}_{2}$ & Swan $\left(\mathrm{d}^{3} \Pi-\mathrm{a}^{4} \Pi\right)$ \\
$\mathrm{H}_{2}$ & Lyman $\left(\mathrm{B}^{1} \Sigma-\mathrm{X}^{1} \Sigma\right)$ \\
$\mathrm{H}_{2}$ & Werner $\left(\mathrm{C}^{1} \Pi-\mathrm{X}^{1} \Sigma\right)$ \\
$\mathrm{H}_{2}$ & $\mathrm{~B} \Sigma-\mathrm{X}^{1} \Sigma$ \\
\hline \hline
\end{tabular}

The temperatures and species concentrations used as inputs NEQAIR are presented in Fig. 9, for the 42 second trajectory point. The spectral emission is presented in Fig. 10. The emission lines for the air species closely match the values measured by the Echelle instrument. However, the calculated CN emission, in the 350 to $450 \mathrm{~nm}$ region, is too high by one order of magnitude. This difference is probably caused by imprecision in the $\mathrm{CN}+\mathrm{O} \rightleftharpoons \mathrm{CO}+\mathrm{N}$ kinetic rate. This discrepancy needs to be further investigated.

\section{Conclusion}

As part of a continuing project to improve heat transfer and ablation rate modeling for hypersonic reentry vehicles, a comprehensive chemistry model for computing the flow around a re-entry vehicle using an ablative heat shield has been tested in a CFD code. The species used in the model were chosen with careful 


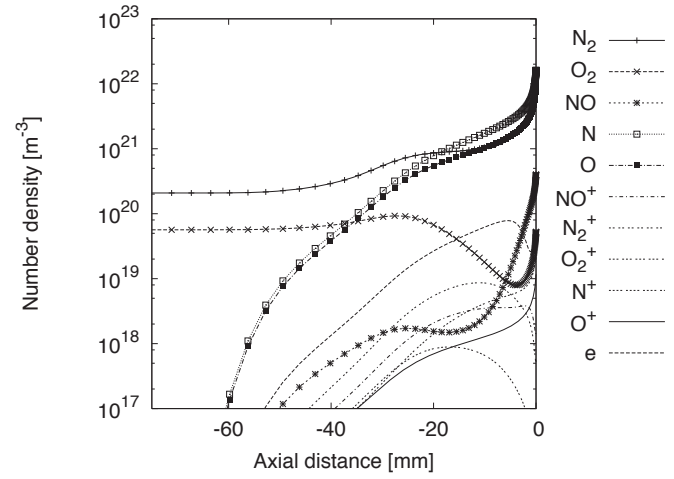

(a) Air species

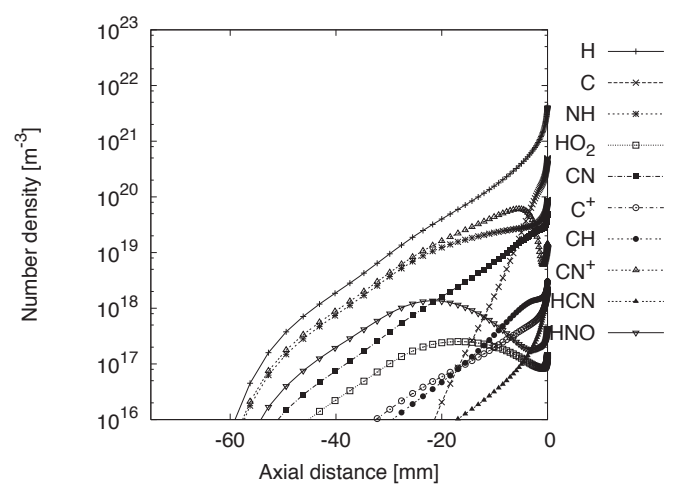

(c) High concentration species

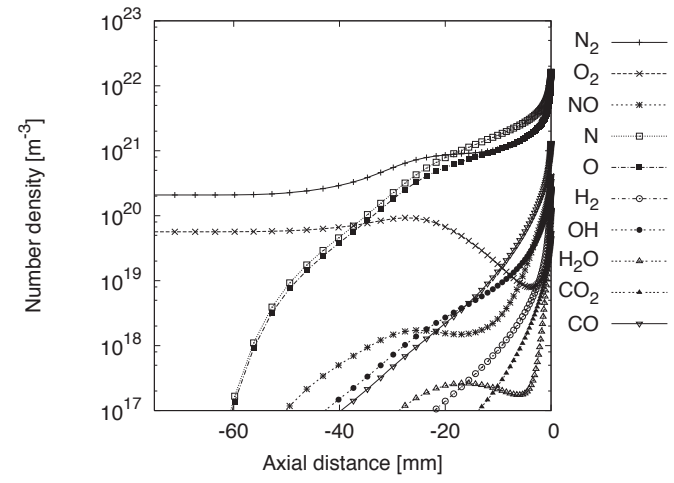

(b) Surface blowing species

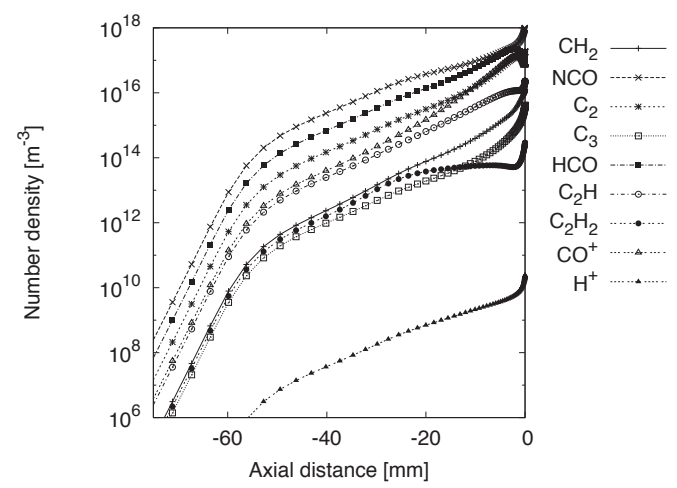

(d) Low concentration species

Figure 6. Species concentrations along the stagnation line for the Stardust re-entry vehicle at $34 \mathrm{~s}$ into re-entry 


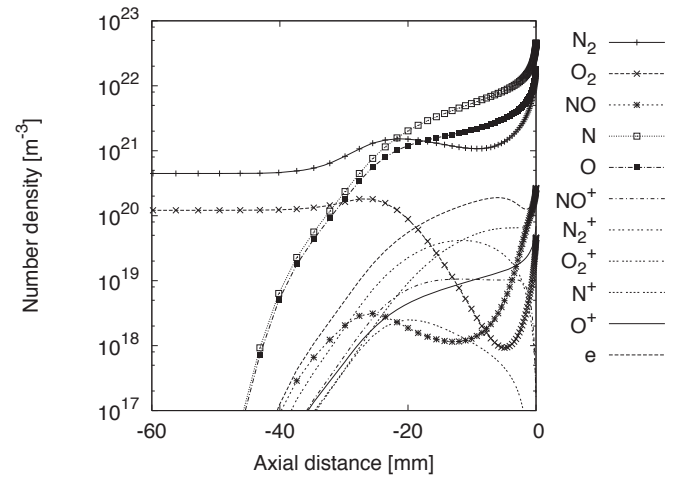

(a) Air species

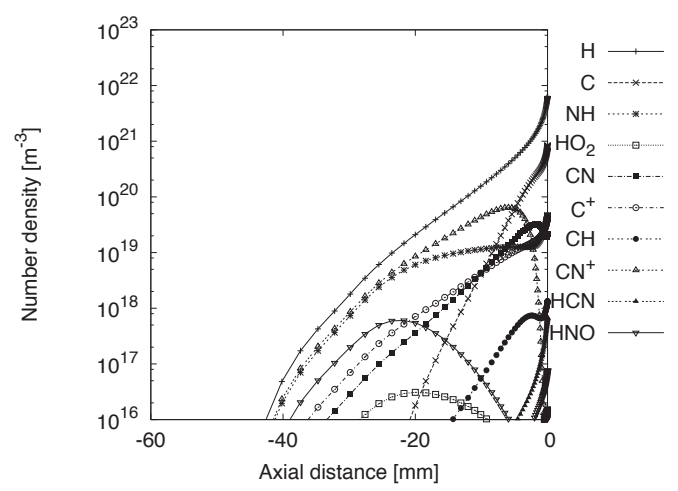

(c) High concentration species

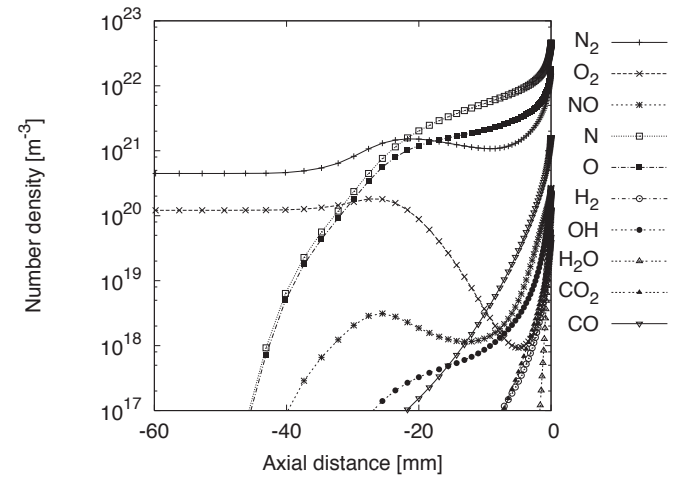

(b) Surface blowing species

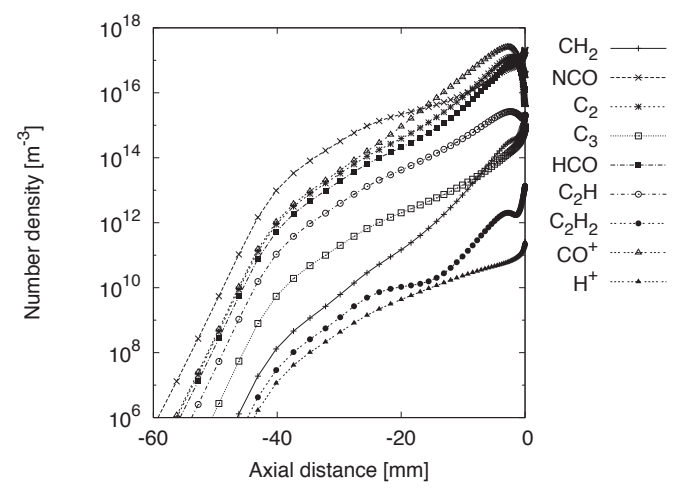

(d) Low concentration species

Figure 7. Species concentrations along the stagnation line for the Stardust re-entry vehicle at $38 \mathrm{~s}$ into re-entry 


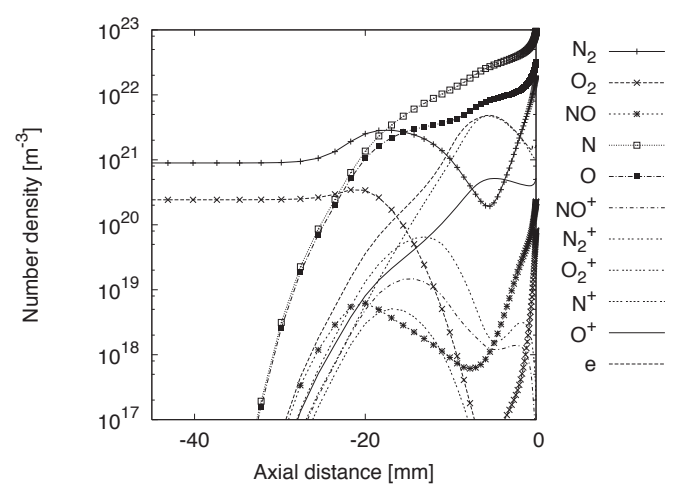

(a) Air species

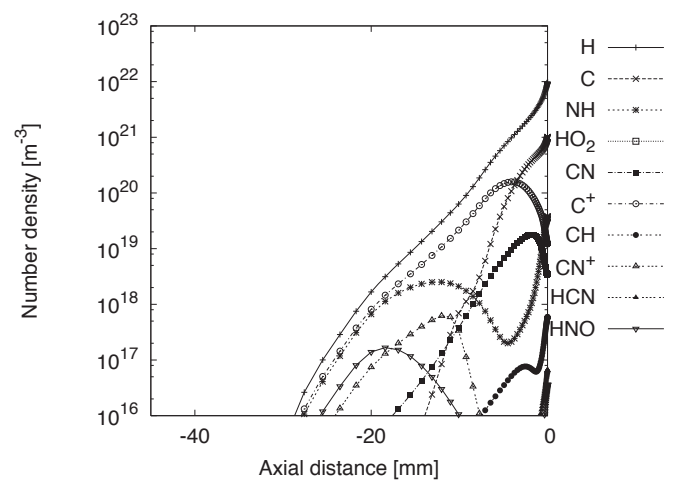

(c) High concentration species

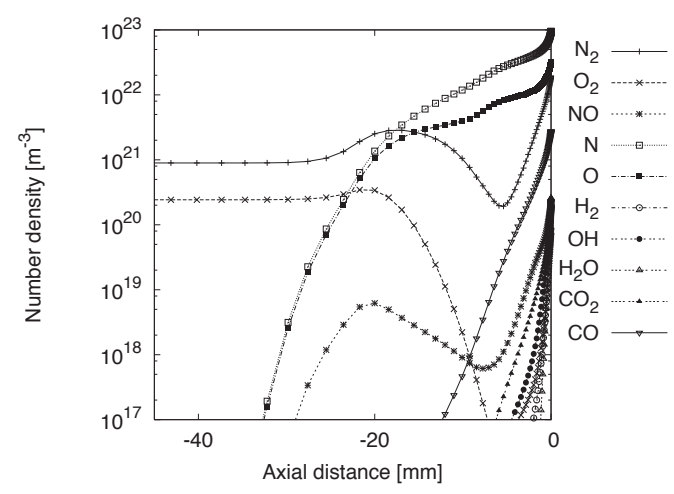

(b) Surface blowing species

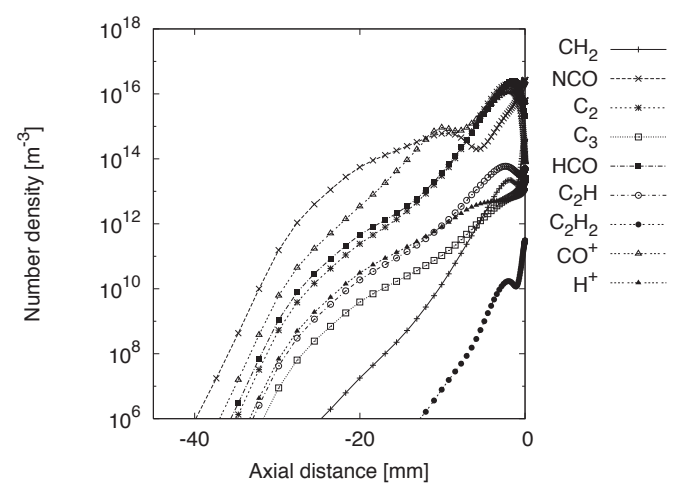

(d) Low concentration species

Figure 8. Species concentrations along the stagnation line for the Stardust re-entry vehicle at $42 \mathrm{~s}$ into re-entry

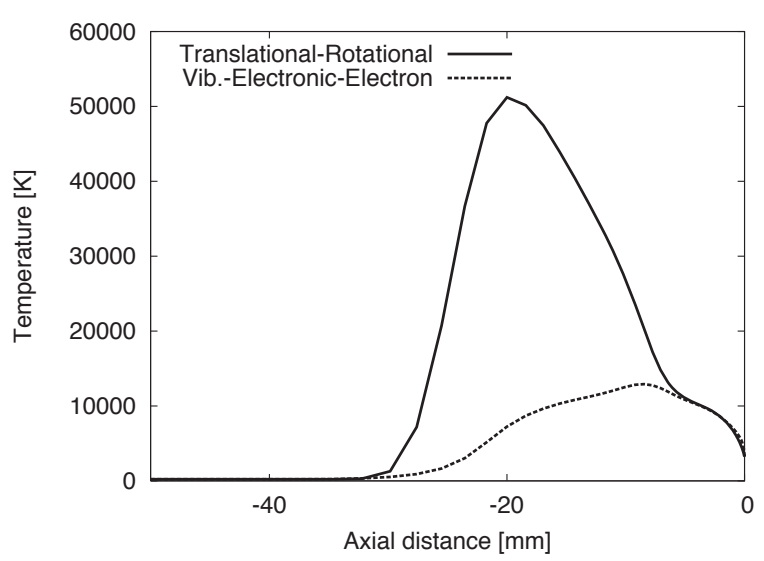

(a) Temperatures

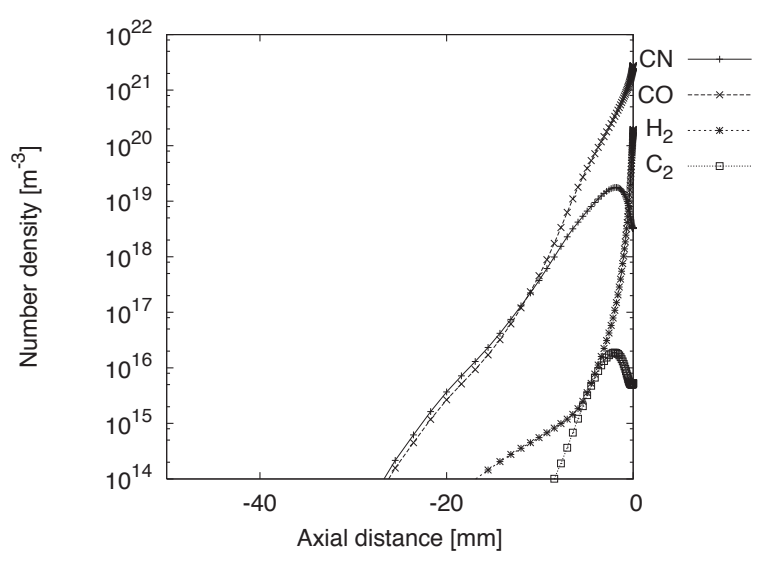

(b) Radiating species considered in NEQAIR

Figure 9. Temperatures and radiating species concentrations along the stagnation line for the Stardust re-entry vehicle at $42 \mathrm{~s}$ into re-entry $(71 \mathrm{~km})$ 


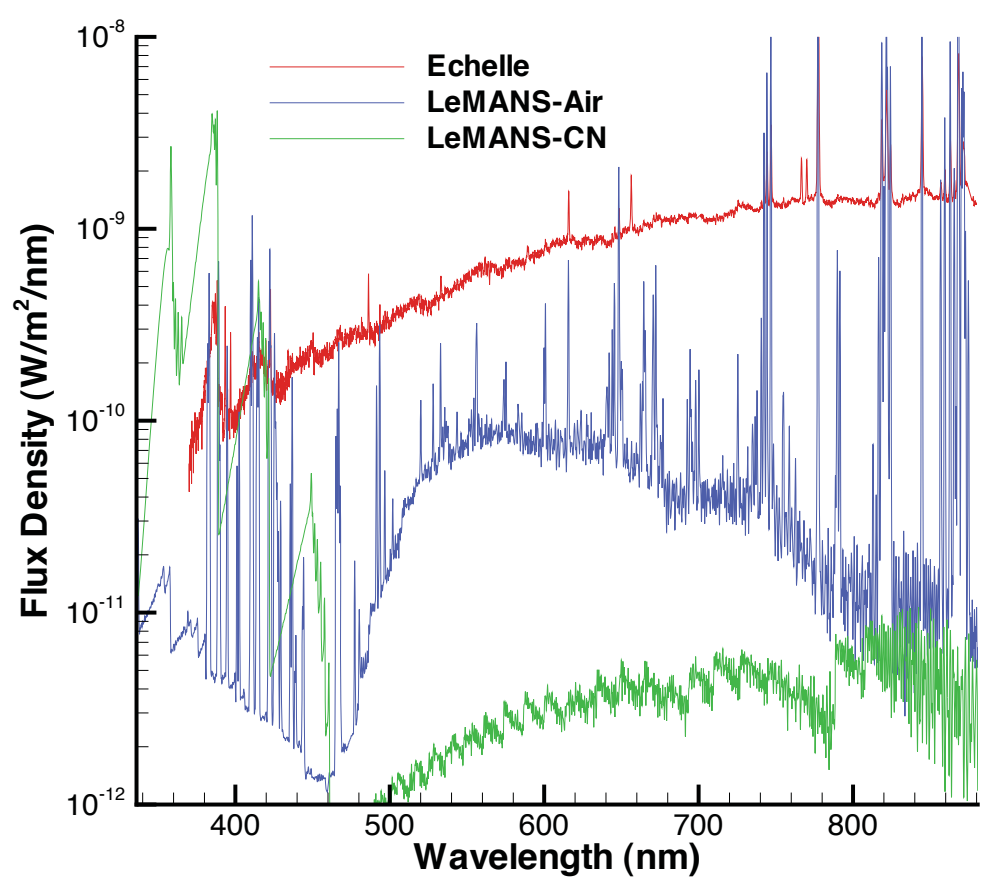

Figure 10. Spectral emission for the Startdust re-entry vehicle at 42 seconds $(71 \mathrm{~km})$

consideration of the flow physics, and the reactions were evaluated and chosen from various sources in the literature. The model was then reduced to a more manageable number of species and reactions, using a zero-dimensional sensitivity analysis, and was validated using various experimental results. Next, the model was implemented in the hypersonic non-equilibrium CFD code LeMANS, and was tested using trajectory points of the continuum regime. These points were chosen because experimental data was available in the form of spectral radiative emission gathered by the Echelle instrument. As expected, the convective heat flux predicted using the new model was significantly reduced relative to the prediction obtained using the equilibrium radiative boundary conditions. The species concentrations along the stagnation line were also presented and it was shown that most species blown from the surface, at chemical equilibrium conditions, immediately react in the flowfield and are transformed. These results clearly indicate the need to use an appropriate chemistry model in the flow field, one that should be significantly different than that assumed in the TPS. It also confirms that the equilibrium assumption at the surface allows the creation of species that would not necessarily be present. Finally, the stagnation line results were used to perform a radiative spectral emission analysis using NEQAIR. The result from one trajectory point was then compared to the experimental data obtain by the Echelle instrument. Although the results show good agreement for all the air species, the emission structure from CN shows a significant discrepancy. Future work includes investigation of the cause of this discrepancy in order to assess the influence of the most important CN forming reaction, $\mathrm{CN}+\mathrm{O} \rightleftharpoons \mathrm{CO}+\mathrm{N}$, on the radiative prediction.

\section{Acknowledgments}

Financial support for this work was provided in part by NASA SBIR Phase-1 Award NNX10CC53P as well as through NASA Prime Contract NNA04BC25C to ELORET Corporation. The authors would also like to thank Dr. Mike Wright, Dr. Ioana Cozmuta, Dr. Tahir Gökçen from NASA Ames, and Dr. Erin Farbar at The University of Michigan, for numerous insightful discussions. 


\section{References}

${ }^{1}$ Suzuki, T., Sawada, K., Yamada, T., and Inatani, Y., "Experimental and Numerical Study of Pyrolysis Gas Pressure in Ablating Test Piece," Journal of Thermophysics and Heat Transfer, Vol. 19, No. 3, July-September 2005, pp. 266-272.

${ }^{2}$ Dec, J. A. and Braun, R. D., "An Approximate Ablative Thermal Protection System Sizing Tool for Entry System Design," 44th AIAA Aerospace Sciences Meeting and Exhibit, No. AIAA 2006-780, Reno, NV, 9-12 January 2006.

${ }^{3}$ Ayasoufi, A., Rahmani, R. K., Cheng, G., Koomullil, R., and Neroorkar, K., "Numerical Simulation of Ablation for Reentry Vehicles," 9th AIAA/ASME Joint Thermophysics and Heat Transfer Conference, No. AIAA 2006-2908, San Francisco, CA, June 5-8 2006.

${ }^{4}$ Amar, A. J., Blackwell, B. F., and Edward, J. R., "One-Dimensional Ablation with Pyrolysis Gas Flow Using a Full Newton's Method and Finite Control Volume Procedure," 39th AIAA Thermophysics Conference, No. AIAA-2007-4535, Miami, FL, 25-28 June 2007, p. 41.

${ }^{5}$ Milos, F. and Chen, Y.-K., "Two-Dimensional Ablation, Thermal Response and Sizing Program for Pyrolyzing Ablators," 46th AIAA Aerospace Sciences Meeting and Exhibit, AIAA-2008-1223, Reno, NV, Jan. 7-10 2008, p. 7.

${ }^{6}$ Venkatachari, B. S., C.Cheng, G., P.Koomullil, R., and Ayasoufi, A., "Computational Tools for Re-entry Aerothermodynamics - Part II. Surface Ablation," 46th AIAA Aerospace Sciences Meeting and Exhibit, AIAA-2008-1218, Reno, NV, Jan. 7-10 2008.

${ }^{7}$ Martin, A. and Boyd, I. D., "Simulation of pyrolysis gas within a thermal protection system," 40th AIAA Thermophysics Conference, No. AIAA-2008-3805, Seattle, WA, June 23-26 2008, p. 20.

${ }^{8}$ Park, C., Jaffe, R. L., and Partridge, H., "Chemical-Kinetic Parameters of Hyperbolic Earth Entry," Journal of Thermophysics and Heat Transfer, Vol. 15, No. 1, January- March 2001, pp. 76-90.

${ }^{9}$ Suzuki, K., Kubota, H., Fujita, K., and Abe, T., "Chemical nonequilibrium ablation analysis of MUSES-C super-orbital reentry capsule," 32nd AIAA Thermophysics Conference, No. AIAA-1997-2481, June 23-25 1997.

${ }^{10}$ Olynick, D., Chen, Y.-K., and Tauber, M. E., "Aerothermodynamics of the Stardust Sample Return Capsule," Journal of Spacecraft and Rockets, Vol. 36, No. 3, May-June 1999, pp. 442-462.

${ }^{11}$ Martin, A., Boyd, I. D., Cozmuta, I., and Wright, M. J., "Chemistry model for ablating carbon-phenolic material during atmospheric re-entry," 48th AIAA Aerospace Sciences Meeting and Exhibit, No. AIAA-2010-1175, Orlando, FL, January 2010.

${ }^{12}$ Martin, A. and Boyd, I. D., "Assessment of carbon-phenolic-in-air chemistry models for atmospheric re-entry," 10th AIAA/ASME Joint Thermophysics and Heat Transfer Conference, No. AIAA-2010-4656, Chicago, IL, June 28th to July 1st 2010.

${ }^{13}$ Whiting, E. E., Park, C., Liu, Y., Arnold, J. O., and Paterson, J. A., "NEQAIR96, Nonequilibrium and Equilibrium Radiative Transport and Spectra Program: User's Manual," Reference Publication 1389, NASA, December 1996.

${ }^{14}$ Wright, M. J., Candler, G. V., and Bose, D., "Data-Parallel Line Relaxation method for the Navier-Stokes equations," AIAA Journal, Vol. 36, No. 9, September 1998, pp. 1603-1609.

${ }^{15}$ Chen, Y.-K. and Milos, F. S., "Ablation and Thermal Response Program for Spacecraft Heatshield Analysis," Journal of Spacecraft and Rockets, Vol. 36, No. 3, May-June 1999, pp. 475-483.

${ }^{16}$ Kinney, D. J., "Aerothermal Anchoring of CBAero using High Fidelity CFD," 45th AIAA Aerospace Sciences Meeting and Exhibit, No. AIAA-2007-0608, Reno, NV, January 8-11 2007.

${ }^{17}$ Milos, F. S. and Chen, Y.-K., "Comprehensive model for multicomponent ablation thermochemistry," 35th Aerospace Sciences Meeting and Exhibit, No. AIAA-1997-141, Reno, NV, Jan. 6-9 1997, p. 9.

${ }^{18}$ Smith, G. P., Golden, D. M., Frenklach, M., Moriarty, N. W., Eiteneer, B., Goldenberg, M., Bowman, C. T., Hanson, R. K., Song, S., William C. Gardiner, J., Lissianski, V. V., and Qin, Z., "GRI-Mech 3.0," 2009.

${ }^{19}$ Gökçen, T., "N ${ }_{2}-\mathrm{CH}_{4}$-Ar Chemical Kinetic Model for Simulations of Titan Atmospheric Entry," Journal of Thermophysics and Heat Transfer, Vol. 21, No. 1, January-March 2007, pp. 9-18.

${ }^{20}$ Kee, R. J., Rupley, F. M., Miller, J. A., Coltrin, M. E., Grcar, J. F., Meeks, E., Moffat, H. K., Lutz, A. E., Dixon-Lewis, G., Smooke, M. D., Warnatz, J., Evans, G. H., Larson, R. S., Mitchell, R. E., Petzold, L. R., Reynolds, W. C., Caracotsios, M., Stewart, W. E., Glarborg, P., Wang, C., McLellan, C. L., Adigun, O., Houf, W., Chou, C. P., Miller, S. F., Ho, P., Young, P. D., Young, D. J., Hodgson, D. W., Petrova, M. V., and Puduppakkam, K. V., "CHEMKIN, Release 4.1," 2006.

${ }^{21}$ Dean, A. J., Davidson, D. F., and Hanson, R. K., "A Shock Tube Study of Reactions of C Atoms with $\mathrm{H}_{2}$ and $\mathrm{O}_{2}$ Using Excimer Photolysis of $\mathrm{C}_{3} \mathrm{O}_{2}$ and C Atom Atomic Resonance Absorption Spectroscopy," The Journal of Physical Chemistry, Vol. 95, No. 1, 1991, pp. 183-191.

${ }^{22}$ Baulch, D. L., Cobos, C. J., Cox, R. A., Frank, P., Hayman, G., Just, T., Kerr, J. A., Murrells, T., Pilling, M. J., Troe, J., Walker, R. W., and Warnatz, J., "Evaluated Kinetic Data for Combustion Modeling. Supplement I," Journal of Physical and Chemical Reference Data, Vol. 23, No. 6, 1994, pp. 847-848.

${ }^{23}$ Andersson, S., Markovic, N., and Nyman, G., "Computational Studies of the Kinetics of the C $+\mathrm{NO}$ and $\mathrm{O}+\mathrm{CN}$ Reactions," J. Phys. Chem. A, Vol. 107, 2003, pp. 5439-5447.

${ }^{24}$ Kruse, T. and Roth, P., "Kinetics of $\mathrm{C}_{2}$ Reactions During High-Temperature Pyrolysis of Acetylene," Journal of Physical Chemistry A, Vol. 101, No. 11, 1997, pp. 2138-2146.

${ }^{25}$ Sommer, T., Kruse, T., and Roth, P., "Perturbation Study on the Reaction of $\mathrm{C}_{2}$ with $\mathrm{N}_{2}$ in High-Temperature $\mathrm{C}_{6} 0 / \mathrm{Ar}$ $+\mathrm{N}_{2}$ Mixtures," Journal of Physical Chemistry A, Vol. 101, No. 20, 1997, pp. 3720- 3725.

${ }^{26}$ Dean, A. J. and Hanson, R. K., "CH and C-Atom Time Histories in Dilute Hydrocarbon Pyrolysis: Measurements and Kinetic Calculations," International Journal of Chemical Kinetics, Vol. 24, No. 6, 1992, pp. 517-532.

${ }^{27}$ Dean, A. J., Hanson, R. K., and Bowman, C. T., "High Temperature Shock Tube Study of Reactions of CH and CAtoms with $\mathrm{N}_{2}$," Twenty- Third Symposium (International) on Combustion, edited by T. C. Institute, Pittsburgh, PA, 1990, pp. 259-265.

${ }^{28}$ Bauerle, S., Klatt, M., and Wagner, H. G., "Recombination and decomposition of methylene radicals at high temperatures," Ber. Bunsenges. Phys. Chem., Vol. 99, 1995, pp. $870-879$. 
${ }^{29}$ Bohland, T., Dobe, S., Temps, F., and Wagner, H., "Kinetics of the reactions between $\mathrm{CH}_{2}\left(\mathrm{X}^{3} \mathrm{~B}_{1}\right)$-radicals and saturated hydrocarbons in the temperature range $296 \mathrm{~K}-707 \mathrm{~K}, "$ Ber. Bunsenges. Phys. Chem., Vol. 89, 1985.

${ }^{30}$ Baulch, D. L., Cobos, C. J., Cox, R. A., Esser, C., Frank, P., Just, T., Kerr, J. A., Pilling, M. J., Troe, J., Walker, R. W., and Warnatz, J., "Evaluated Kinetic Data for Combustion Modelling," Journal of Physical and Chemical Reference Data, Vol. 21, No. 3, 1992, pp. 411-734.

${ }^{31}$ Sanders, W., Lin, C., and Lin, M., "Short communication on the importance of the reaction $\mathrm{CH}_{2}+\mathrm{N}_{2}=\mathrm{HCN}+\mathrm{NH}$ as a precursor for prompt NO formation," Combust. Sci. Technol., Vol. 51, 1987, pp. 103 - 108.

${ }^{32}$ Tsang, W. and Hampson, R., "Chemical kinetic data base for combustion chemistry. Part I. Methane and related compounds," J. Phys. Chem. Ref. Data, Vol. 15, 1986.

${ }^{33}$ Park, C., Howe, J. T., Jaffe, R. L., and Candler, G. V., "Review of Chemical Kinetic Problems of Future NASA Missions, II: Mars Entries," Journal of Thermophysics and Heat Transfer, Vol. 8, No. 1, March 1994, pp. 9-23.

${ }^{34}$ Davidson, D. F., Dean, A. J., Dirosa, M. D., and Hanson, R. K., "Shock tube measurements of the reactions of CN with $\mathrm{O}$ and $\mathrm{O}_{2}, "$ International Journal of Chemical Kinetics, Vol. 23, No. 11, 1991, pp. 1035-1050.

${ }^{35}$ Lindackers, D., Burmeister, M., and Roth, P., "High-Temperature Kinetics of the Reaction CN $+\mathrm{CO}_{2}$," Combustion and Flame, Vol. 81, No. 3-4, 1990, pp. 251-259.

${ }^{36}$ Caridade, P., Rodrigues, S., Sousa, F., and Varandas, A., "Unimolecular and bimolecular calculations for HN 2 ," J. Phys. Chem. A, Vol. 109, 2005, pp. $2356-2363$.

${ }^{37}$ Tsang, W. and Herron, J. T., "Chemical Kinetic Data Base for Propellant Combustion 1. Reactions Involving NO, NO2 , HNO, HNO2, HCN and N2O," Journal of Physical Chemistry Reference Data, Vol. 20, No. 4, 1991, pp. 609-663.

${ }^{38}$ Park, C., Nonequilibrium Hypersonic Aerothermodynamics, Wiley-Interscience, February 1990.

${ }^{39}$ Baulch, D. L., Bowman, C. T., Cobos, C. J., Cox, R. A., Just, T., Kerr, J. A., Pilling, M. J., Stocker, D., Troe, J., Tsang, W., Walker, R. W., and Warnatz, J., "Evaluated Kinetic Data for Combustion Modeling: Supplement II," Journal of Physical and Chemical Reference Data, Vol. 34, No. 3, 2005, pp. 757-1397.

${ }^{40}$ Dean, A. J., Hanson, R. K., and Bowman, C. T., "A Shock Tube Study of Reactions of C Atoms and CH with NO Including Product Channel Measurements," Journal of Chemical Physics, Vol. 95, 1991, pp. 3180-3189.

${ }^{41}$ Mick, H.-J. and Roth, P., "High Temperature Thermal Decomposition of CO and CN," Shock Waves, edited by SpringerVerlag, Berlin, 1992, p. 805.

${ }^{42}$ Dean, A. J. and Hanson, R. K., "CH and C-atom time histories in dilute hydrocarbon pyrolysis: Measurements and kinetics calculations," International Journal of Chemical Kinetics, Vol. 24, No. 6, 2004, pp. 517 - 532.

${ }^{43}$ Scalabrin, L. C. and Boyd, I. D., "Numerical Simulations of the FIRE-II Convective and Radiative Heating Rates," 39th AIAA Thermophysics Conference, No. AIAA-2007-4044, Miami, FL, 25 - 28 June 2007, p. 17.

${ }^{44}$ Scalabrin, L. C., Numerical Simulation of Weakly Ionized Hypersonic Flow Over Reentry Capsules., Ph.D. thesis, The University of Michigan, Ann Arbor, MI, 2007.

${ }^{45}$ Scalabrin, L. C. and Boyd, I. D., "Development of an Unstructured Navier-Stokes Solver for Hypersonic Nonequilibrium Aerothermodynamics," 38th AIAA Thermophysics Conference, No. AIAA-2005-5203, Toronto, Ontario, June 6-9 2005, pp. $1-18$.

${ }^{46}$ Scalabrin, L. C. and Boyd, I. D., "Numerical Simulation of Weakly Ionized Hypersonic Flow for Reentry Configurations," 9th AIAA/ASME Joint Thermophysics and Heat Transfer Conference, No. AIAA-2006-3773, San Francisco, CA, June 5-8 2006, p. 18.

${ }^{47}$ Holman, J. P., Heat transfer, Mc Graw-Hill : Publishing Company, 1990.

${ }^{48}$ Gnoffo, P. A., "Upwind-Biased, Point-implicit Relaxation Strategies for Viscous Hypersonic Flows," 9th AIAA Computational Fluid Dynamics Conference, No. AIAA-1989-1972-CP, Buffalo, NY, June 13-15 1989, pp. 415-425.

${ }^{49}$ Martinelli, S. and Ruffin, S., "Validation Process for Blowing and Transpiration-Cooling in DPLR," 39th AIAA Thermophysics Conference, No. AIAA-2007-4255, 2007, p. 9.

${ }^{50}$ Thompson, R. A. and Gnoffo, P. A., "Implementation of a Blowing Boundary Condition in the LAURA Code," 46th AIAA Aerospace Sciences Meeting and Exhibit, No. AIAA-2008-1243, Reno, NV, Jan. 7-10 2008, p. 11.

${ }^{51}$ Martin, A. and Boyd, I. D., "Implicit implementation of material response and moving meshes for hypersonic re-entry ablation," 47th AIAA Aerospace Sciences Meeting and Exhibit, No. AIAA-2009-0670, Orlando, FL, Jan. 5-8 2009.

${ }^{52}$ Trumble, K. A., Cozmuta, I., Sepka, S., and Jenniskens, P., "Post-flight Aerothermal Analysis of the Stardust Sample Return Capsule," 46th AIAA Aerospace Sciences Meeting and Exhibit, AIAA-2008-1201, Reno, NV, Jan. 7-10 2008. 